\title{
Adaptive Computational Methods for Parabolic Problems
}

\author{
K. Eriksson, C. Johnson and A. Logg \\ Department of Computational Mathematics, Chalmers University of Technology, \\ SE-412 96 Göteborg, Sweden
}

\begin{abstract}
We present a unified methodology for the computational solution of parabolic systems of differential equations with adaptive selection of discretization in space and time, based on a posteriori error estimates involving residuals of computed solutions and stability factors/weights, obtained by solving an associated linearized dual problem. We define parabolicity as boundedness in time (up to logarithmic factors) of a certain strong stability factor measuring the $L_{1}\left(L_{2}\right)$-norm in time-space of the time derivative of the dual solution with $L_{2}$-normalized initial data.

KEY WORDS: parabolic, finite elements, stiff, space-time discretization, Galerkin method, convection-diffusion-reaction, stability factor/weight, dual linearized problem, adaptive error control, time step control, a priori error estimate, a posteriori error estimate
\end{abstract}

The simpler a hypothesis is, the better it is. (Leibniz)

1. What is a parabolic problem?

A common classification of partial differential equations uses the terms elliptic, parabolic and hyperbolic, with the stationary Poisson equation being a prototype example of an elliptic problem, the time-dependent heat equation that of a parabolic problem, and the time-dependent wave equation being a hyperbolic problem. More generally, parabolic problems are often described vaguely speaking as "diffusiondominated", while hyperbolic problems are "convection-dominated" in a setting of systems of convection-diffusion equations. Alternatively, the term "stiff problems" is used to describe parabolic problems, with the term stiff referring to the characteristic presence of a range of time scales, varying from slow to fast with increasing damping.

In the context of computational methods for a general class of systems of timedependent convection-diffusion-reaction equations, the notion of "parabolicity" or "stiffness" may be given a precise quantitative definition, which will be at the 
focal point of this presentation. We will define a system of convection-diffusionreaction equations to be parabolic if computational solution is possible over long time without error accumulation, or alternatively, if a certain strong stability factor $S_{c}(T)$, measuring error accumulation, is of unit size independent of the length $T$ in time of the simulation. More precisely, the error accumulation concerns the Galerkin discretization error in a discontinuous Galerkin method $\mathrm{dG}(q)$ with piecewise polynomials of degree $q$ of order $2 q+1$. (The total discretization error may also contain a quadrature error, which typically accumulates at a linear rate in time for a parabolic problem.) This gives parabolicity a precise quantitative meaning with a direct connection to computational methods. A parabolic problem thus exhibits a feature of "loss of memory" for Galerkin errors satisfying an orthogonality condition, which allows long-time integration without error accumulation. As shall be made explicit below, our definition of parabolicity through a certain stability factor is closely related to the definition of an analytic semigroup.

For a typical hyperbolic problem the corresponding strong stability factor will grow linearly in time, while for more general initial value problems the growth may be polynomial or even exponential in time.

The solutions of parabolic systems in general vary considerably in space-time and from one component to the other with occasional transients where derivatives are large. Efficient computational methods for parabolic problems thus require adaptive control of the mesh size in both space space and time, or more general multi-adaptive control with possibly different resolution in time for different components.

\section{Outline}

We first consider in Section 3 time-stepping methods for Initial Value Problems (IVPs) for systems of ordinary differential equations. We present an a posteriori error analysis exhibiting the characteristic feature of a parabolic problem of nonaccumulation of Galerkin errors in the setting of the backward Euler method (the discontinuous Galerkin method $\mathrm{dG}(0)$ ), with piecewise constant (polynomial of order 0 ) approximation in time. The a posteriori error estimate involves the residual of the computed solution and stability factors/weights obtained by solving an associated dual linearized problem expressing in quantitative form the stability features of the IVP being solved. The a posteriori error estimate forms the basis of an adaptive method for time step control with the objective of controlling the Euclidean norm of the error uniformly in time or at selected time levels, or some other output quantity. The form of the a posteriori error estimate expresses the characteristic feature of a parabolic problem that the time step control is independent of the length in time of the simulation.

In Section 4 we compute stability factors for a couple of IVPs modeling chemical reactions and find that the strong stability factor $S_{c}(T)$ remains of unit size over long time. 
In Section 5 we contrast with an IVP with exponentially growing stability factors: the Lorenz system.

The backward Euler method, or more generally the $\mathrm{dG}(q)$ method, is implicit and requires the solution of a nonlinear system of equations at each time step. In Section 6 we study iterative fixed point-type solution strategies resembling explicit timestepping methods. However, since explicit time-stepping for stiff problems is unstable unless the time step is smaller than the fastest time scale, which may be unnecessarily restrictive outside fast transients, we include a stabilization technique based on adaptively stabilizing the stiff system by taking a couple of small time steps when needed. We show efficiency gain factors compared to traditional explicit methods with the time step restriction indicated, of the order 10-100 or more depending on the problem. The need for explicit-type methods for parabolic problems avoiding forming Jacobians and solving associated linear systems of equations, is very apparent for the large systems of convection-diffusion-reaction equations arising in the modeling of chemical reactors with many reactants and reactions involved. The need for explicit time-stepping also arises in the setting of multi-adaptive time-stepping with the time step varying in both space and for different reactants, since here the discrete equations may be coupled over several time steps for some of the subdomains (or reactants), leading to very large systems of algebraic equations.

In Section 7 we prove the basic strong stability estimates for an abstract parabolic model problem, and connect to the definition of an analytic semigroup.

In Sections 8-15 we present adaptive space-time Galerkin finite element methods for a model parabolic IVP, the heat equation, including a priori and a posteriori error estimates. The space-time Galerkin discretization method cG $(p) \mathrm{dG}(q)$ is based on the continuous Galerkin method $\mathrm{cG}(p)$ with piecewise polynomials of degree $p$ in space, and the discontinuous Galerkin method $\mathrm{dG}(q)$ with piecewise polynomials of degree $q$ in time (for $q=0,1$ ). In Section 16 we discuss briefly the extension to convectiondiffusion-reaction systems, and present computational results in Section 17.

\section{Introduction to adaptive methods for IVPs}

We now give a brief introduction to the general topic of adaptive error control for numerical time-stepping methods for initial value problems, with special reference to parabolic or stiff problems. In an adaptive method, the time steps are chosen automatically with the purpose of controlling the numerical error to within a given tolerance level. The adaptive method is based on an a posteriori error estimate involving the residual of the computed solution and results of auxiliary computations of stability factors, or more generally stability weights.

We consider an IVP of the form

$$
\dot{u}(t)=f(u(t)) \text { for } 0<t \leq T, \quad u(0)=u^{0},
$$

where $f: \mathbb{R}^{d} \rightarrow \mathbb{R}^{d}$ is a given differentiable function, $u^{0} \in \mathbb{R}^{d}$ a given initial value, and $T>0$ a given final time. For the computational solution of (1), we let 
$0=t_{0}<t_{1}<\ldots<t_{n-1}<t_{n}<\ldots<t_{N}=T$ be an increasing sequence of discrete time steps with corresponding time intervals $I_{n}=\left(t_{n-1}, t_{n}\right]$ and time steps $k_{n}=t_{n}-t_{n-1}$, and consider the backward Euler method: Find $U\left(t_{n}\right)$ successively for $n=0,1, \ldots, N$, according to the formula

$$
U\left(t_{n}\right)=U\left(t_{n-1}\right)+k_{n} f\left(U\left(t_{n}\right)\right)
$$

with $U(0)=u^{0}$. The backward Euler method is implicit in the sense that to compute the value $U\left(t_{n}\right)$ with $U\left(t_{n-1}\right)$ already computed, we need to solve a system of of equations. We will return to this aspect below.

We associate a function $U(t)$ defined on $[0, T]$ to the function values $U\left(t_{n}\right)$, $n=0,1, \ldots, N$, as follows:

$$
U(t)=U\left(t_{n}\right) \quad \text { for } t \in\left(t_{n-1}, t_{n}\right]
$$

In other words, $U(t)$ is left-continuous piecewise constant on $[0, T]$ and takes the value $U\left(t_{n}\right)$ on $I_{n}$, and thus takes a jump from the limit from the left $U\left(t_{n-1}^{-}\right)=U\left(t_{n-1}\right)$ to the limit from the right $U\left(t_{n-1}^{+}\right)=U\left(t_{n}\right)$ at the time level $t=t_{n-1}$. We can now write the backward Euler method in the form

$$
U\left(t_{n}\right)=U\left(t_{n-1}\right)+\int_{t_{n-1}}^{t_{n}} f(U(t)) d t
$$

or equivalently

$$
U\left(t_{n}\right) \cdot v=U\left(t_{n-1}\right) \cdot v+\int_{t_{n-1}}^{t_{n}} f(U(t)) \cdot v d t,
$$

for all $v \in \mathbb{R}^{d}$ with the dot signifying the scalar product in $\mathbb{R}^{d}$. This method is also referred to as $\mathrm{dG}(0)$, the discontinuous Galerkin method of order zero, corresponding to approximating the exact solution by a piecewise constant function $U(t)$ satisfying the orthogonality condition (3).

The general $\mathrm{dG}(q)$ method takes the form (3), with the restriction to each time interval $I_{n}$ of the solution $U(t)$ and the test function $v$ on each time interval $I_{n}$ being polynomial of degree $q$. The $\mathrm{dG}(q)$ method comes also in multi-adaptive form with each component and corresponding test function being piecewise polynomial with possibly different sequences of time steps for different components.

We shall now derive an a posteriori error estimate, aiming at control of the scalar product of the error $e(T)=(u-U)(T)$ at final time $T$ with a given vector $\psi$, where we assume that $\psi$ is normalized so that $\|\psi\|=1$. We introduce the following linearized dual problem running backward in time:

$$
-\dot{\phi}(t)=A^{\top}(t) \phi(t) \quad \text { for } 0 \leq t<T, \quad \phi(T)=\psi,
$$

with

$$
A(t)=\int_{0}^{1} f^{\prime}(s u(t)+(1-s) U(t)) d s
$$


where $u(t)$ is the exact solution and $U(t)$ the approximate solution, $f^{\prime}$ is the Jacobian of $f$, and $\top$ denotes transpose. We note that $f(u(t))-f(U(t))=A(t)(u(t)-U(t))$. We now start from the identity

$$
e(T) \cdot \psi=e(T) \cdot \psi+\sum_{n=1}^{N} \int_{t_{n-1}}^{t_{n}} e \cdot\left(-\dot{\phi}-A^{\top} \phi\right) d t
$$

and integrate by parts on each subinterval $\left(t_{n-1}, t_{n}\right)$ to get the error representation:

$$
e(T) \cdot \psi=\sum_{n=1}^{N} \int_{t_{n-1}}^{t_{n}}(\dot{e}-A e) \cdot \phi d t-\sum_{n=1}^{N}\left(U\left(t_{n}\right)-U\left(t_{n-1}\right)\right) \cdot \phi\left(t_{n-1}\right)
$$

where the last term results from the jumps of $U(t)$ at the the nodes $t=t_{n-1}$. Since now $u$ solves the differential equation $\dot{u}-f(u)=0$, and $\dot{U}=0$ on each time interval $\left(t_{n-1}, t_{n}\right)$, we have

$$
\dot{e}-A e=\dot{u}-f(u)-\dot{U}+f(U)=-\dot{U}+f(U)=f(U) \quad \text { on }\left(t_{n-1}, t_{n}\right) .
$$

It follows that

$$
e(T) \cdot \psi=-\sum_{n=1}^{N}\left(U\left(t_{n}\right)-U\left(t_{n-1}\right)\right) \cdot \phi\left(t_{n-1}\right)+\int_{0}^{T} f(U) \cdot \phi d t
$$

Using (3) with $v=\bar{\phi}_{n}$, the mean value of $\phi$ over $I_{n}$, we get

$e(T) \cdot \psi=-\sum_{n=1}^{N}\left(U\left(t_{n}\right)-U\left(t_{n-1}\right)\right) \cdot\left(\phi\left(t_{n-1}\right)-\bar{\phi}_{n}\right)+\sum_{n=1}^{N} \int_{t_{n-1}}^{t_{n}} f(U) \cdot\left(\phi-\bar{\phi}_{n}\right) d t$.

Since now

$$
\int_{t_{n-1}}^{t_{n}} f(U)\left(\phi-\bar{\phi}_{n}\right) d t=0
$$

because $f(U(t))$ is constant on $\left(t_{n-1}, t_{n}\right]$, the error representation takes the form

$$
e(T) \cdot \psi=-\sum_{n=1}^{N}\left(U\left(t_{n}\right)-U\left(t_{n-1}\right)\right) \cdot\left(\phi\left(t_{n-1}\right)-\bar{\phi}_{n}\right) .
$$

Finally, from the estimate

$$
\left.\| \phi\left(t_{n-1}\right)-\bar{\phi}_{n}\right)\left\|\leq \int_{t_{n-1}}^{t_{n}}\right\| \dot{\phi}(t) \| d t
$$

where $\|\cdot\|$ denotes the the Euclidean norm in $\mathbb{R}^{d}$, we obtain the following a posteriori error estimate for the backward Euler or $\mathrm{dG}(0)$ method:

$$
|e(T) \cdot \psi| \leq S_{c}(T, \psi) \max _{1 \leq n \leq N}\left\|U\left(t_{n}\right)-U\left(t_{n-1}\right)\right\|
$$

Encyclopedia of Computational Mechanics. Edited by Erwin Stein, René de Borst and Thomas J.R. Hughes. (c) 2004 John Wiley \& Sons, Ltd. 
where the stability factor $S_{c}(T, \psi)$ is defined by

$$
S_{c}(T, \psi)=\int_{0}^{T}\|\dot{\phi}(t)\| d t .
$$

Maximizing over $\psi$ with $\|\psi\|=1$, we obtain a posteriori control of the Euclidean norm of $e(T)$ :

$$
\left.\left.\|e(T)\| \leq S_{c}(T) \max _{1 \leq n \leq N} \| U\left(t_{n}\right)\right)-U\left(t_{n-1}\right)\right) \|,
$$

with corresponding stability factor

$$
S_{c}(T)=\max _{\|\psi\|=1} S_{c}(T, \psi) .
$$

Equivalently, we can write this estimate as

$$
\|e(T)\| \leq S_{c}(T) \max _{0 \leq t \leq T}\|k(t) R(U(t))\|,
$$

where $k(t)=k_{n}=t_{n}-t_{n-1}$ for $t \in\left(t_{n-1}, t_{n}\right]$, and $R(U(t))=\left(U\left(t_{n}\right)-\right.$ $\left.U\left(t_{n-1}\right)\right) / k_{n}=f\left(U\left(t_{n}\right)\right)$ corresponds to the residual obtained by inserting the discrete solution into the differential equation (noting that $\dot{U}(t)=0$ on each time interval).

We can express the a posteriori error estimate (5) alternatively in the form

$$
|e(T) \cdot \psi| \leq \int_{0}^{T} k(t) R(U(t))\|\dot{\phi}(t)\| d t
$$

where now the dual solution enters as a weight in a time integral involving the residual $R(U(t))$. Maximizing over $k(t) R(U(t))$ and integrating $\|\dot{\phi}(t)\|$ we obtain the original estimate (9).

We now define the IVP (1) to be parabolic if (up to possibly logarithmic factors) the stability factor $S_{c}(T)$ is of unit size for all $T$. We shall see that another typical feature of a parabolic problem is that the stability factor $S_{c}(T, \psi)$ varies little with the specific choice of normalized initial data $\psi$, which means that to compute $S_{c}(T)=$ $\max _{\|\psi\|=1} S_{c}(T, \psi)$, we may drastically restrict the variation $\psi$ and solve the dual problem with only a few different initial data.

If we perturb $f$ to $\hat{f}$ in the discretization with $\mathrm{dG}(q)$, for instance by approximating $f(U(t))$ by a polynomial connecting to quadrature in computing $\int_{I_{n}} f(U(t)) d t$, we obtain an additional contribution to the a posteriori error estimate of the form

$$
S_{q}(T, \psi) \max _{[0, T]}\|f(U(t))-\hat{f}(U(t))\|,
$$

or $S_{q}(T) \max _{[0, T]}\|f(U(t))-\hat{f}(U(t))\|$, with corresponding stability factors defined by

$$
S_{q}(T, \psi)=\int_{0}^{T}\|\phi(t)\| d t
$$

Encyclopedia of Computational Mechanics. Edited by Erwin Stein, René de Borst and Thomas J.R. Hughes.

(c) 2004 John Wiley \& Sons, Ltd. 
where $\phi$ solves the backward dual problem with $\phi(T)=\psi$, and $S_{q}(T)=$ $\max _{\|\psi\|=1} S_{q}(T, \psi)$. In a parabolic problem we may have $S_{q}(T) \sim T$, although $S_{c}(T) \sim 1$ for all $T>0$. We note that $S_{c}(T)$ involves the time derivative $\dot{\phi}$, while $S_{q}(T)$ involves the dual $\phi$ itself.

Note that in $\mathrm{dG}(0)$ there is no need for quadrature in the present case of an autonomous IVP, since then $f(U(t))$ is piecewise constant. However, in a corresponding non-autonomous problem of the form $\dot{u}=f(u(t), t)$ with $f$ depending explicitly on $t$, quadrature may be needed also for $\mathrm{dG}(0)$.

The basic parabolic or stiff problem is a linear constant coefficient IVP of the form $\dot{u}(t)=f(u(t), t) \equiv-A u(t)+f(t)$ for $0<t \leq T, u(0)=u^{0}$, with $A$ a constant positive semidefinite symmetric matrix with eigenvalues ranging from small to large positive. In this case, $f^{\prime}(u)=-A$ with eigenvalues $\lambda \geq 0$ and corresponding solution components varying on time scales $1 / \lambda$ ranging from very long (slow variation/decay if $\lambda$ is small positive) to very short (fast variation/decay if $\lambda$ is large positive). A solution to a typical stiff problem thus has a range of time-scales varying from slow to fast. In this case the dual problem takes the form $-\dot{\phi}(t)=-A \phi(t)$ for $0 \leq t<T$, and the strong stability estimate states that, independent of the distribution of the eigenvalues $\lambda \geq 0$ of $A$, we have

$$
\int_{0}^{T}(T-t)\|\dot{\phi}(t)\|^{2} d t \leq \frac{1}{4},
$$

where we assume that $\|\phi(T)\|=1$. From this we may derive that for $0<\epsilon<T$,

$$
\int_{0}^{T-\epsilon}\|\dot{\phi}(t)\| d t \leq \frac{1}{2}(\log (T / \epsilon))^{1 / 2},
$$

which up to a logarithmic factor states that $S_{c}(T) \sim 1$ for all $T>0$. Further, the corresponding (weak) stability estimate states that $\|\phi(t)\| \leq\|\psi\|$, from which directly follows that that $S_{q}(T) \leq T$ as indicated. The (simple) proofs of the stability estimates are given below.

The stability factors $S_{c}(T, \psi)$ and $S_{q}(T, \psi)$ may be approximately computed a posteriori by replacing $A(t)$ in (4) with $f^{\prime}(U(t))$, assuming $U(t)$ is sufficiently close to $u(t)$ for all $t$, and solving the corresponding backward dual problem numerically (e.g. using the $\mathrm{dG}(0)$ method). We may similarly compute approximations of $S_{c}(T)$ and $S_{q}(T)$ by varying $\psi$. By computing the stability factors we get concrete evidence of the parabolicity of the underlying problem, which may be difficult (or impossible) to assess analytically a priori. Of course, there is also a gradual degeneracy of the parabolicity as the stability factor $S_{c}(T)$ increases.

A special feature of many parabolic problems is that $S_{c}(T, \psi)$ varies little with the specific choice of initial data, which makes it possible to compute $S_{c}(T)$ by solving the dual problem a few times with different initial data and taking the maximum. We give a simple motivation for this below.

The a posteriori error estimate (7) can be used as the basis for an adaptive time- 
stepping algorithm, controlling the size of the Galerkin discretization error, of the form: For $n=1,2, \ldots, N$, choose $k_{n}$ so that

$$
\left.\left.\| U\left(t_{n}\right)\right)-U\left(t_{n-1}\right)\right) \| \approx \frac{\mathrm{TOL}}{S_{c}(T)},
$$

for some tolerance TOL $>0$. Recalling that the characteristic feature of a parabolic problem is that $S_{c}(T) \sim 1$ for all $T>0$, this means that the time step control related to the Galerkin discretization error will be independent of the length of the time interval of the simulation. This means that long-time integration without error accumulation is possible, which may be interpreted as some kind of "parabolic loss of memory". We note again that this concerns the Galerkin error only, which has this special feature as a consequence of the Galerkin orthogonality. However, the quadrature error may accumulate in time typically at a linear rate, and so a long-time simulation may require more accurate quadrature than a simulation over a shorter interval.

\section{Examples of stiff IVPs}

We have stated above that a parabolic or stiff initial value problem $\dot{u}(t)=f(u(t))$ for $0<t \leq T, u(0)=u^{0}$, may be characterized by the fact that the stability factor $S_{c}(T)$ is of moderate (unit) size independent of $T>0$, while the norm of the linearized operator $f^{\prime}(u(t))$ may be large, corresponding to the presence of large negative eigenvalues. Such initial value problems are common in models of chemical reactions, with reactions on a range of time scales varying from slow to fast. Typical solutions include so-called transients where the fast reactions make the solution change quickly over a short (initial) time interval, after which the fast reactions are "burned out" and the slow reactions make the solution change on a longer time scale. We now consider a set of test problems which we solve by the adaptive $\mathrm{dG}(0)$ method, including computation of the strong stability factor $S_{c}(T)$.

\subsection{Model problem: $\dot{u}+A u(t)=f(t)$ with A positive symmetric semidefinite}

As indicated, the basic example of a parabolic IVP takes the form $\dot{u}+A u(t)=f(t)$ for $0<t \leq T, u(0)=u^{0}$, where $A$ is a positive semidefinite square matrix. We consider here the case

$$
A \approx\left(\begin{array}{rrrrr}
-4.94 & 2.60 & 0.11 & 0.10 & 0.06 \\
2.60 & -4.83 & 2.69 & 0.17 & 0.10 \\
0.11 & 2.69 & -4.78 & 2.69 & 0.11 \\
0.10 & 0.17 & 2.69 & -4.83 & 2.60 \\
0.06 & 0.10 & 0.11 & 2.60 & -4.94
\end{array}\right)
$$

with eigenvalues $(0,-2.5,-5,-7.5,-9.33)$. In Figure 1 , we plot the solution, a dual solution and the stability factor $S_{c}(T, \psi)$ as a function of $T$ for a collection of different initial values $\phi(T)=\psi$. We note that the variation with $\psi$ is rather small: about a factor 4 . 

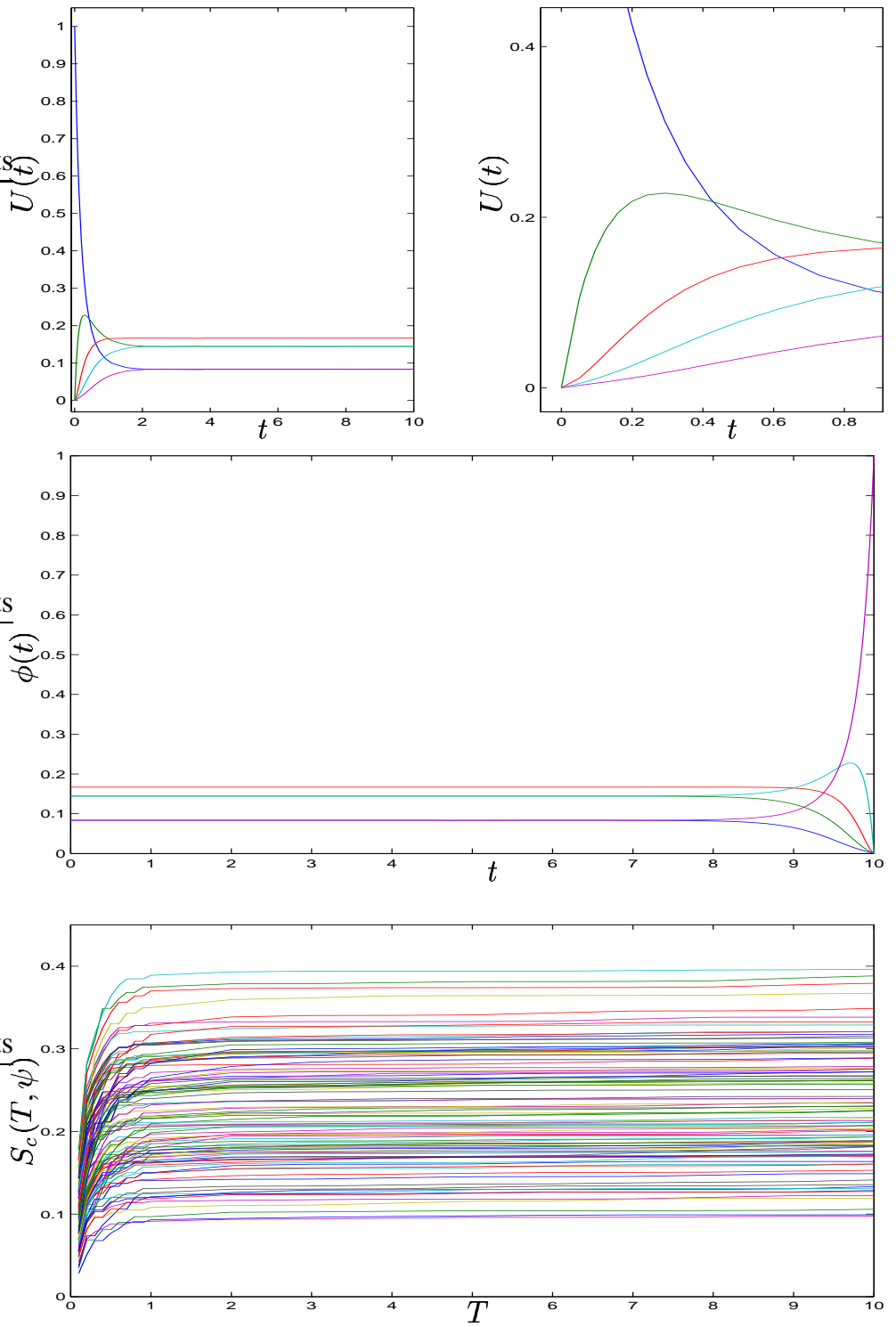

Figure 1. Symmetric IVP: solution, dual solution and stability factors $S_{c}(T, \psi)$. 


\subsection{The Akzo-Nobel system of chemical reactions}

We next consider the so-called Akzo-Nobel problem, which is a test problem for solvers of stiff ODEs modeling chemical reactions: Find the concentrations $u(t)=$ $\left(u_{1}(t), \ldots, u_{6}(t)\right)$ such that for $0<t \leq T$,

$$
\left\{\begin{array}{l}
\dot{u}_{1}=-2 r_{1}+r_{2}-r_{3}-r_{4}, \\
\dot{u}_{2}=-0.5 r_{1}-r_{4}-0.5 r_{5}+F \\
\dot{u}_{3}=r_{1}-r_{2}+r_{3} \\
\dot{u}_{4}=-r_{2}+r_{3}-2 r_{4} \\
\dot{u}_{5}=r_{2}-r_{3}+r_{5} \\
\dot{u}_{6}=-r_{5}
\end{array}\right.
$$

where $F=3.3 \cdot\left(0.9 / 737-u_{2}\right)$ and the reaction rates are given by $r_{1}=18.7 \cdot u_{1}^{4} \sqrt{u_{2}}$, $r_{2}=0.58 \cdot u_{3} u_{4}, r_{3}=0.58 / 34.4 \cdot u_{1} u_{5}, r_{4}=0.09 \cdot u_{1} u_{4}^{2}$ and $r_{5}=0.42 \cdot u_{6}^{2} \sqrt{u_{2}}$, with the initial condition $u^{0}=(0.437,0.00123,0,0,0,0.367)$. In Figure 2 we plot the solution, a dual solution and the stability factor $S_{c}(T)$ as a function of $T$. We note the initial transients in the concentrations and their long-time very slow variation after the active phase of reaction. We also note that $S_{c}(T)$ initially grows to about 3.5 and then falls back to a value around 2. This is a typical behavior for reactive systems, where momentarily during the active phase of reaction the perturbation growth may be considerable, while over long-time the memory of that phase fades. On the other hand $S_{q}(T)$ grows consistently, which shows that fading memory requires some mean-value to be zero (Galerkin orthogonality). We present below more examples of this nature exhibiting features of parabolicity.

\section{A non-stiff IVP: the Lorenz system}

The Lorenz system presented 1972 by the meteorologist Edward Lorenz:

$$
\left\{\begin{array}{l}
\dot{u_{1}}=-10 u_{1}+10 u_{2}, \\
\dot{u_{2}}=28 u_{1}-u_{2}-u_{1} u_{3}, \\
\dot{u_{3}}=-\frac{8}{3} u_{3}+u_{1} u_{2}, \\
u(0)=u^{0}
\end{array}\right.
$$

is an example of an IVP with exponentially growing stability factors reflecting a strong sensitivity to perturbations. Lorenz chose the model to illustrate perturbation sensitivity in meteorological models, making forecasts of daily weather virtually impossible over a period of more than a week. For the Lorenz system accurate numerical solution using double precision beyond 50 units of time seems impossible. Evidently, the Lorenz system is not parabolic.

The system (12) has three equilibrium points $\bar{u}$ with $f(\bar{u})=0: \bar{u}=(0,0,0)$ and $\bar{u}=( \pm 6 \sqrt{2}, \pm 6 \sqrt{2}, 27)$. The equilibrium point $\bar{u}=(0,0,0)$ is unstable with the corresponding Jacobian $f^{\prime}(\bar{u})$ having one positive (unstable) eigenvalue and two 

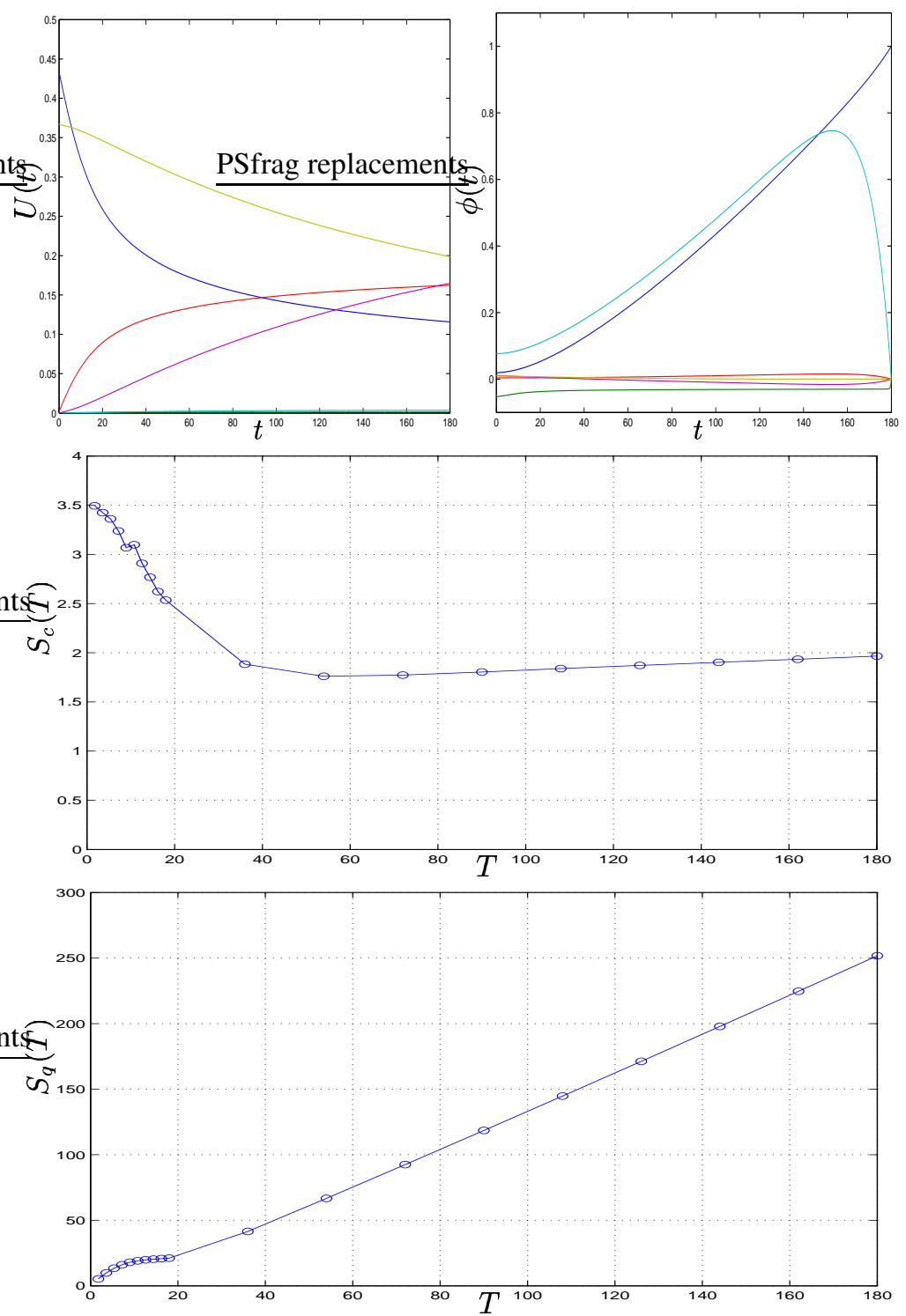

Figure 2. The Akzo-Nobel problem: solution, dual solution, stability factor $S_{c}(T, \psi)$, and stability factor $S_{q}(T, \psi)$. 
negative (stable) eigenvalues. The equilibrium points $( \pm 6 \sqrt{2}, \pm 6 \sqrt{2}, 27)$ are slightly unstable with the corresponding Jacobians having one negative (stable) eigenvalue and two eigenvalues with very small positive real part (slightly unstable) and also an imaginary part. More precisely, the eigenvalues at the two non-zero equilibrium points are $\lambda_{1} \approx-13.9$ and $\lambda_{2,3} \approx .0939 \pm 10.1 i$.

In Figure 3, we present two views of a solution $u(t)$ that starts at $u(0)=(1,0,0)$ computed to time 30 with an error tolerance of TOL $=0.5$ using an adaptive IVPsolver of the form presented above. The plotted trajectory is typical: it is kicked away from the unstable point $(0,0,0)$ and moves towards one of the non-zero equilibrium points. It then slowly orbits away from that point and at some time decides to cross over towards the other non-zero equilibrium point, again slowly orbiting away from that point and coming back again, orbiting out, crossing over, and so on. This pattern of some orbits around one non-zero equilibrium point followed by a transition to the other non-zero equilibrium point is repeated with a seemingly random number of revolutions around each non-zero equilibrium point.
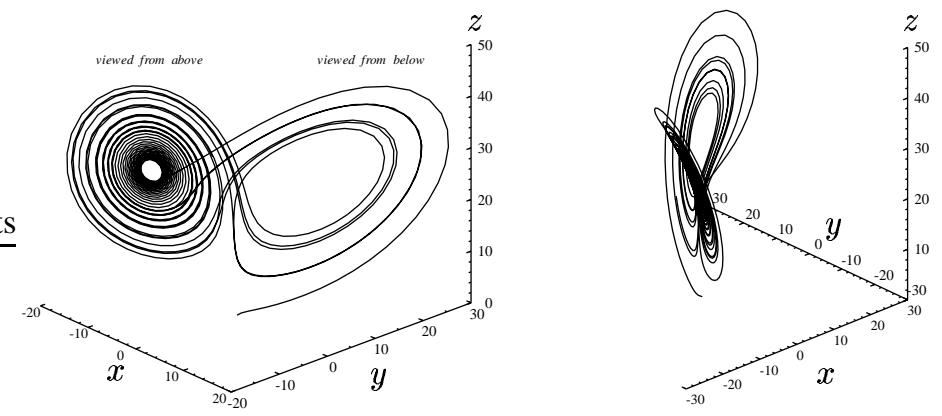

Figure 3. Two views of a numerical trajectory of the Lorenz system over the time interval $[0,30]$.

In Figure 4, we plot the size of the stability factor $S_{q}(T)$ connected to quadrature errors as function of final time $T$. We notice that the stability factor takes an exponential leap every time the trajectory flips, while the growth is slower when the trajectory orbits one of the non-zero equilibrium points. The stability factor grows on the average as $10^{T / 3}$ which sets the effective time limit of accurate computation to $T \approx 50$ computing in double precision with say 15 accurate digits.

\section{Explicit time-stepping for stiff IVPs}

The $\mathrm{dG}(0)$ method for the IVP $\dot{u}=f(u)$ takes the form

$$
U\left(t_{n}\right)-k_{n} f\left(U\left(t_{n}\right)\right)=U\left(t_{n-1}\right)
$$

Encyclopedia of Computational Mechanics. Edited by Erwin Stein, René de Borst and Thomas J.R. Hughes.

(C) 2004 John Wiley \& Sons, Ltd. 


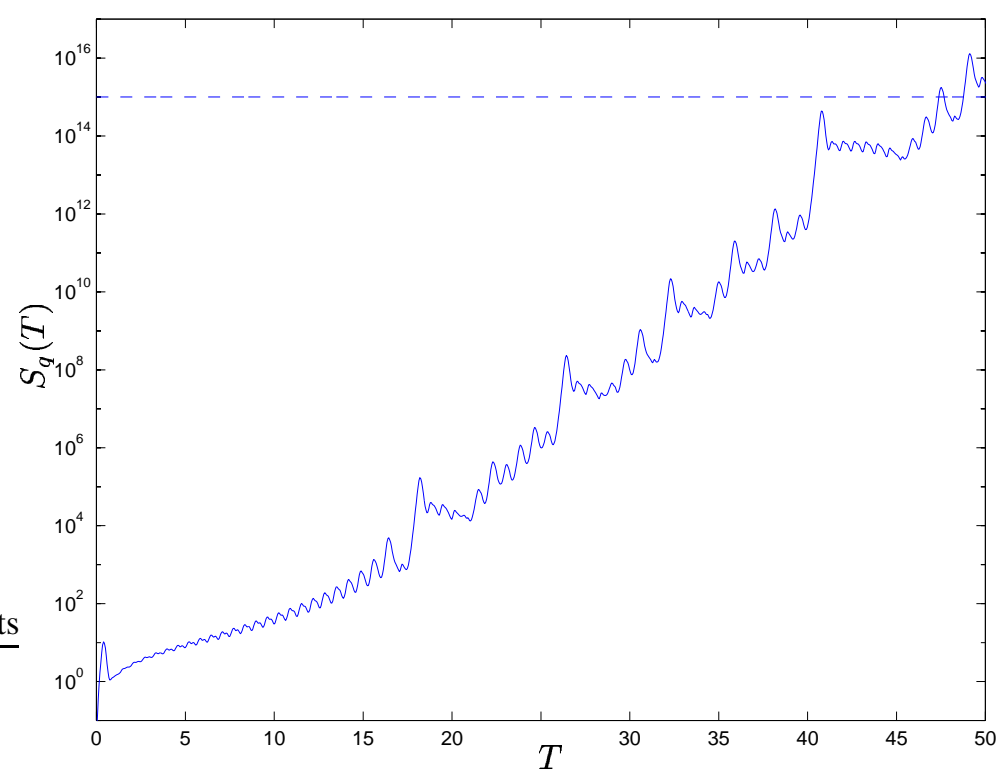

Figure 4 . The growth of the stability factor $S_{q}(T)$ for the Lorenz problem.

At each time step we have to solve an equation of the form $v-k_{n} f(v)=U\left(t_{n-1}\right)$ with $U\left(t_{n-1}\right)$ given. To this end we may try a damped fixed point iteration in the form

$$
v^{(m)}=(I-\alpha) v^{(m-1)}+\alpha\left(U\left(t_{n-1}\right)+k_{n} f\left(v^{(m-1)}\right)\right),
$$

with $\alpha$ some suitable matrix (or constant in the simplest case). Choosing $\alpha=I$ with only one iteration corresponds to the explicit Euler method. Convergence of the fixed point iteration requires that

$$
\left\|I-\alpha+k_{n} \alpha f^{\prime}(v)\right\|<1,
$$

for relevant values of $v$, which could force $\alpha$ to be small (e.g. in the stiff case with $f^{\prime}(v)$ having large negative eigenvalues) and result in slow convergence. A simple choice is to take $\alpha$ to be a diagonal matrix with $\alpha_{i i}=1 /\left(1-k_{n} f_{i i}^{\prime}\left(v^{(m-1)}\right)\right)$, corresponding to a diagonal approximation of Newton's method, with hope that the number of iterations will be small.

We just learned that explicit time-stepping for stiff problems requires small time steps outside transients and thus may be inefficient. We shall now indicate a way to get around this limitation through a process of stabilization, where a large time step is accompanied by a couple of small time steps. The resulting method has similarities with the control system of a modern (unstable) jet fighter like the Swedish JAS Gripen, the flight of which is controlled by quick small flaps of a pair of small extra wings 
ahead of the main wings, or balancing a stick vertically on the finger tips if we want a more domestic application.

We shall now explain the basic (simple) idea of the stabilization and present some examples, as illustrations of fundamental aspects of adaptive IVP-solvers and stiff problems. Thus to start with, suppose we apply the explicit Euler method to the scalar problem

$$
\begin{aligned}
\dot{u}(t)+\lambda u(t) & =0 \quad \text { for } 0<t \leq T, \\
u(0) & =u^{0},
\end{aligned}
$$

with $\lambda>0$ taking first a large time step $K$ satisfying $K \lambda>2$ and then $m$ small time steps $k$ satisfying $k \lambda<2$, to get the method

$$
U\left(t_{n}\right)=(1-k \lambda)^{m}(1-K \lambda) U\left(t_{n-1}\right),
$$

altogether corresponding to a time step of size $k_{n}=K+m k$. Here $K$ gives a large unstable time step with $|1-K \lambda|>1$ and $k$ is a small time step with $|1-k \lambda|<1$. Defining the polynomial function $p(x)=(1-\theta x)^{m}(1-x)$, where $\theta=\frac{k}{K}$, we can write the method (14) in the form

$$
U\left(t_{n}\right)=p(K \lambda) U\left(t_{n-1}\right)
$$

For stability, we need

$$
|p(K \lambda)| \leq 1, \quad \text { that is }|1-k \lambda|^{m}(K \lambda-1) \leq 1,
$$

or

$$
m \geq \frac{\log (K \lambda-1)}{-\log |1-k \lambda|} \approx 2 \log (K \lambda),
$$

with $c=k \lambda \approx 1 / 2$ for definiteness.

We conclude that $m$ may be quite small even if $K \lambda$ is large, since the logarithm grows so slowly, and then only a small fraction of the total time (a small fraction of the time interval $[0, T]$ ) will be spent on stabilizing time-stepping with the small time steps $k$.

To measure the efficiency gain we introduce

$$
\alpha=\frac{1+m}{K+k m} \in(1 / K, 1 / k),
$$

which is the number of time steps per unit time interval with the stabilized explicit Euler method. By (15) we have

$$
\alpha \approx \frac{1+2 \log (K \lambda)}{K+\log (K \lambda) / \lambda} \approx 2 \lambda \frac{\log (K \lambda)}{K \lambda} \ll 2 \lambda,
$$

for $K \lambda \gg 1$. On the other hand, the number of time steps per unit time interval for the standard explicit Euler method is

$$
\alpha_{0}=\lambda / 2
$$

Encyclopedia of Computational Mechanics. Edited by Erwin Stein, René de Borst and Thomas J.R. Hughes. (c) 2004 John Wiley \& Sons, Ltd. 
with the maximum stable time step $k_{n}=2 / \lambda$.

The cost reduction factor using the stabilized explicit Euler method would thus be

$$
\frac{\alpha}{\alpha_{0}} \approx \frac{4 \log (K \lambda)}{K \lambda}
$$

which can be quite significant for large values of $K \lambda$.

We now present some examples using an adaptive cG(1) IVP-solver, where explicit fixed point iteration (using only a couple of iterations) on each time interval is combined with stabilizing small time steps, as described for the explicit Euler method. In all problems we note the initial transient, where the solution components change quickly, and the oscillating nature of the time step sequence outside the transient, with large time steps followed by some small stabilizing time steps.

Example. We apply the indicated method to the scalar problem (13) with $u^{0}=1$ and $\lambda=1000$, and display the result in Figure 5. The cost reduction factor in comparison to a standard explicit method is large: $\alpha / \alpha_{0} \approx 1 / 310$.
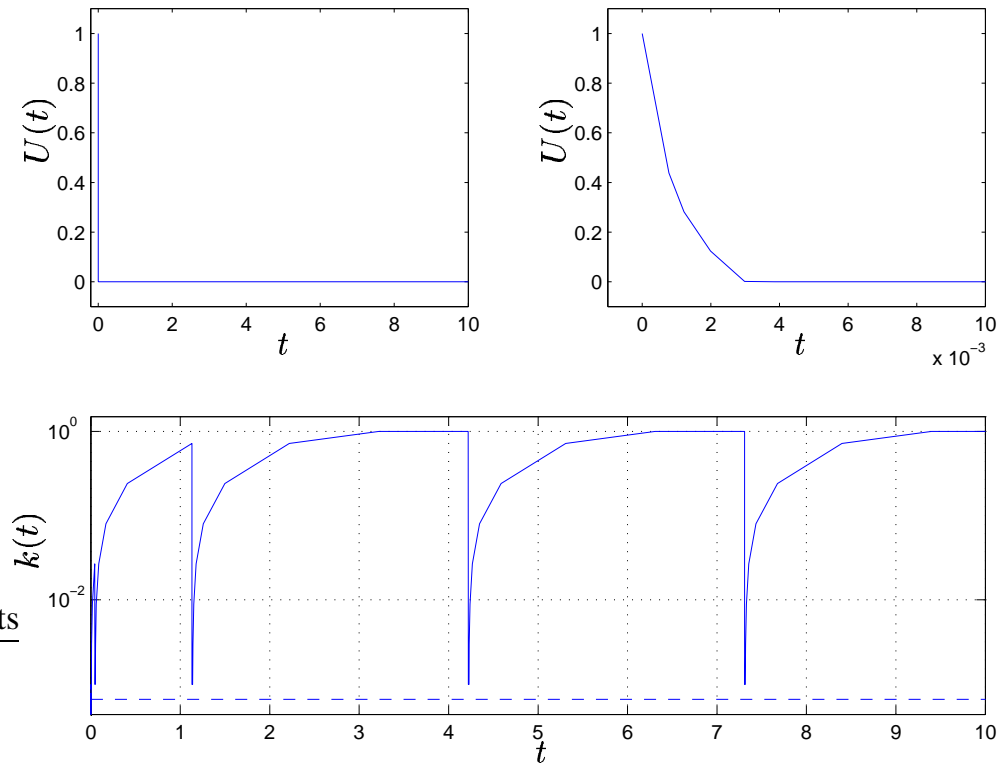

Figure 5. Solution and time step sequence for eq. (13), $\alpha / \alpha_{0} \approx 1 / 310$.

Example. We now consider the $2 \times 2$ diagonal system

$$
\begin{aligned}
\dot{u}(t)+\left(\begin{array}{cc}
100 & 0 \\
0 & 1000
\end{array}\right) u(t) & =0 \quad \text { for } 0<t \leq T, \\
u(0) & =u^{0}
\end{aligned}
$$

Encyclopedia of Computational Mechanics. Edited by Erwin Stein, René de Borst and Thomas J.R. Hughes. (C) 2004 John Wiley \& Sons, Ltd. 
with $u^{0}=(1,1)$. There are now two eigenmodes with large eigenvalues that need to be stabilized. The cost reduction factor is $\alpha / \alpha_{0} \approx 1 / 104$.
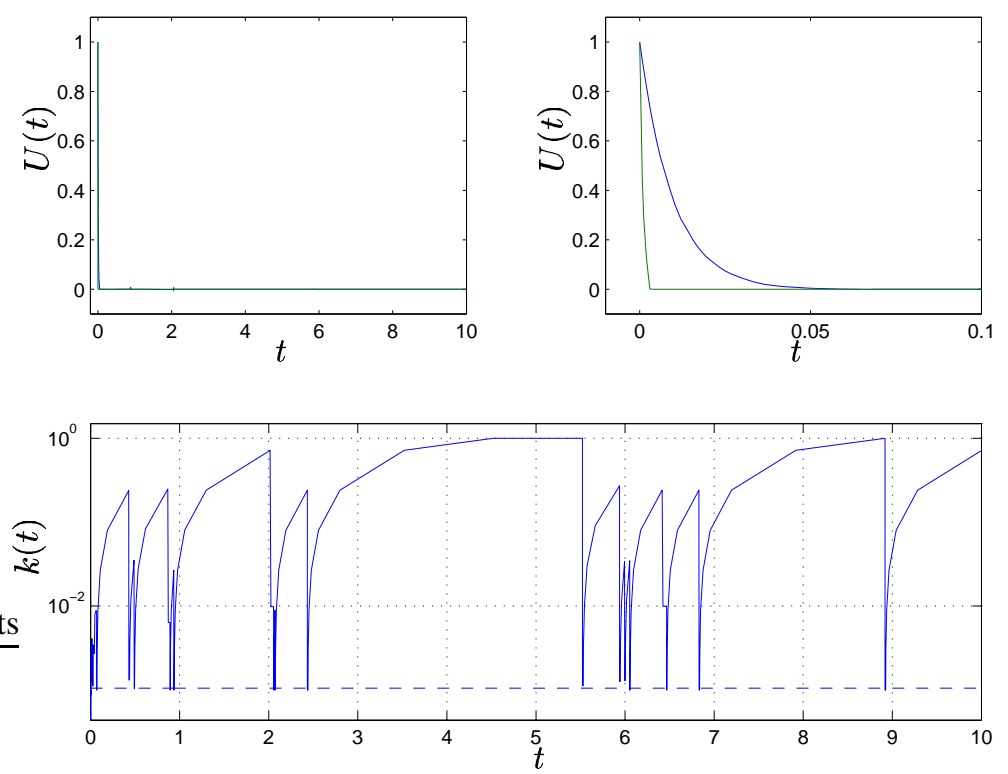

Figure 6. Solution and time step sequence for eq. (18), $\alpha / \alpha_{0} \approx 1 / 104$.

Example. We next consider the so-called HIRES problem ("High Irradiance RESponse") from plant physiology which consists of the following eight equations:

$$
\left\{\begin{array}{l}
\dot{u}_{1}=-1.71 u_{1}+0.43 u_{2}+8.32 u_{3}+0.0007 \\
\dot{u}_{2}=1.71 u_{1}-8.75 u_{2}, \\
\dot{u}_{3}=-10.03 u_{3}+0.43 u_{4}+0.035 u_{5}, \\
\dot{u}_{4}=8.32 u_{2}+1.71 u_{3}-1.12 u_{4}, \\
\dot{u}_{5}=-1.745 u_{5}+0.43 u_{6}+0.43 u_{7}, \\
\dot{u}_{6}=-280.0 u_{6} u_{8}+0.69 u_{4}+1.71 u_{5}-0.43 u_{6}+0.69 u_{7} \\
\dot{u}_{7}=280.0 u_{6} u_{8}-1.81 u_{7} \\
\dot{u}_{8}=-280.0 u_{6} u_{8}+1.81 u_{7},
\end{array}\right.
$$

together with the initial condition $u^{0}=(1.0,0,0,0,0,0,0,0.0057)$. We present the solution and the time step sequence in Figure 7 . The cost is now $\alpha \approx 8$ and the cost reduction factor is $\alpha / \alpha_{0} \approx 1 / 33$.

Example. We consider again the Akzo-Nobel problem from above, integrating over the interval $[0,180]$. We plot the solution and the time step sequence in Figure 8. 

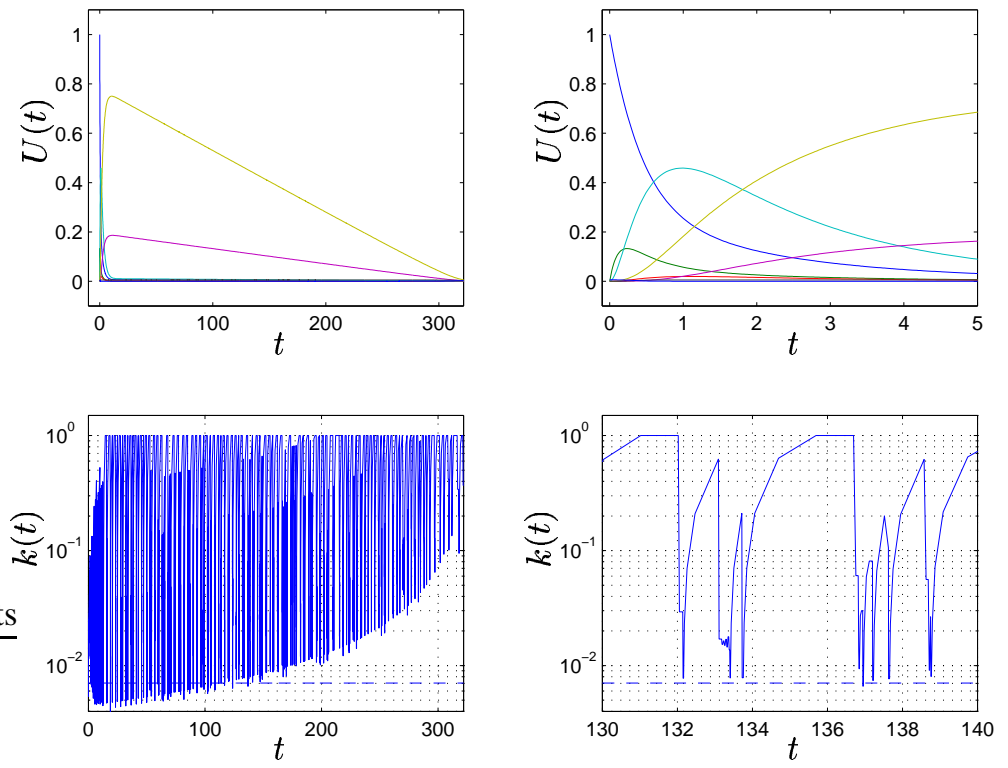

Figure 7. Solution and time step sequence for eq. (19), $\alpha / \alpha_{0} \approx 1 / 33$.

Allowing a maximum time step of $k_{\max }=1$ (chosen arbitrarily), the cost is $\alpha \approx 2$ and the cost reduction factor is $\alpha / \alpha_{0} \approx 1 / 9$. The actual gain in a specific situation is determined by the quotient between the large time steps and the small damping time steps, as well as the number of small damping steps that are needed. In this case the number of small damping steps is small, but the large time steps are not very large compared to the small damping steps. The gain is thus determined both by the stiff nature of the problem and the tolerance (or the size of the maximum allowed time step).

Example. We consider now Van der Pol's equation:

$$
\ddot{u}+\mu\left(u^{2}-1\right) \dot{u}+u=0,
$$

which we write as

$$
\left\{\begin{array}{l}
\dot{u}_{1}=u_{2} \\
\dot{u}_{2}=-\mu\left(u_{1}^{2}-1\right) u_{2}-u_{1}
\end{array}\right.
$$

We take $\mu=1000$ and solve on the interval $[0,10]$ with initial condition $u^{0}=(2,0)$. The cost is now $\alpha \approx 140$ and the cost reduction factor is $\alpha / \alpha_{0} \approx 1 / 75$. 

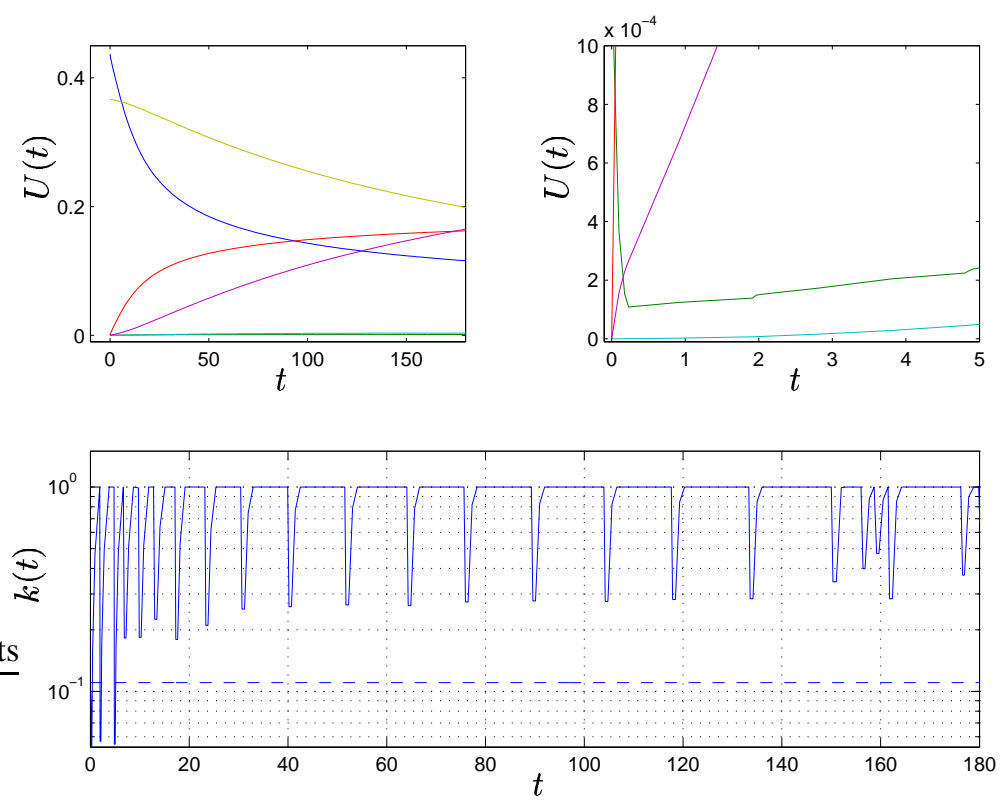

Figure 8. Solution and time step sequence for the Akzo-Nobel problem, $\alpha / \alpha_{0} \approx 1 / 9$.

7. Strong stability estimates for an abstract parabolic model problem

We consider an abstract parabolic model problem of the form: Find $w(t) \in H$ such that

$$
\left\{\begin{array}{l}
\dot{w}(t)+A w(t)=0 \quad \text { for } 0<t \leq T \\
w(0)=w^{0}
\end{array}\right.
$$

where $H$ is a vector space with inner product $(\cdot, \cdot)$ and norm $\|\cdot\|, A$ is a positive semi-definite symmetric linear operator defined on a subspace of $H$, i.e. $A$ is a linear transformation satisfying $(A w, v)=(w, A v)$ and $(A v, v) \geq 0$ for all $v$ and $w$ in the domain of definition of $A$, and $w^{0}$ is the initial data. In the model problem of Section $3, H=\mathbb{R}^{d}$ and $A$ is a positive semi-definite symmetric $d \times d$ matrix. In the case of the heat equation, considered in the next section, $H=L_{2}(\Omega)$ and $-A=\Delta$ (the Laplacian) with homogeneous Dirichlet boundary conditions.

We now state and prove the basic strong stability estimates for the parabolic model problem (21), noting that the constants on the right-hand sides of the estimates are independent of the positive semi-definite symmetric operator $A$. It should be noted that the dual backward problem of (21), $-\dot{\phi}+A \phi=0$, takes the form (21) with $w(t)=\phi(T-t)$. 

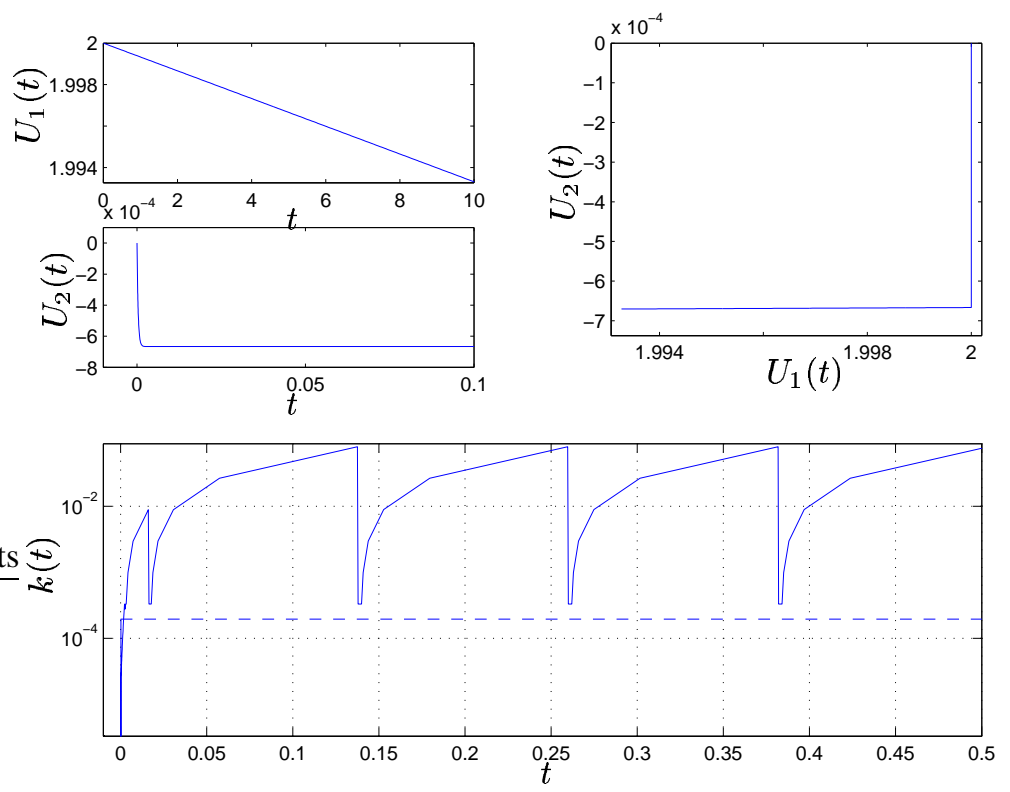

Figure 9. Solution and time step sequence for eq. (20), $\alpha / \alpha_{0} \approx 1 / 75$.

Lemma 7.1. The solution $w$ of (21) satisfies for $T>0$,

$$
\begin{gathered}
\|w(T)\|^{2}+2 \int_{0}^{T}(A w(t), w(t)) d t=\left\|w^{0}\right\|^{2}, \\
\int_{0}^{T} t\|A w(t)\|^{2} d t \leq \frac{1}{4}\left\|w^{0}\right\|^{2} \\
\|A w(T)\| \leq \frac{1}{\sqrt{2} T}\left\|w^{0}\right\| .
\end{gathered}
$$

Proof. Taking the inner product of $\dot{w}(t)+A w(t)=0$ with $w(t)$, we obtain

$$
\frac{1}{2} \frac{d}{d t}\|w(t)\|^{2}+(A w(t), w(t))=0
$$

from which (22) follows.

Next, taking the inner product of $\dot{w}(t)+A w(t)=0$ with $t A w(t)$ and using the fact that

$$
(\dot{w}(t), t A w(t))=\frac{1}{2} \frac{d}{d t}(t(A w(t), w(t)))-\frac{1}{2}(A w(t), w(t)),
$$

since $A$ is symmetric, we find after integration that

$$
\frac{1}{2} T(A w(T), w(T))+\int_{0}^{T} t\|A w(t)\|^{2} d t=\frac{1}{2} \int_{0}^{T}(A w(t), w(t)) d t
$$

Encyclopedia of Computational Mechanics. Edited by Erwin Stein, René de Borst and Thomas J.R. Hughes. (C) 2004 John Wiley \& Sons, Ltd. 
from which (23) follows using (22) and the fact that $(A w, w) \geq 0$.

Finally, taking the inner product in with $t^{2} A^{2} w(t)$, we obtain

$$
\frac{1}{2} \frac{d}{d t}\left(t^{2}\|A w(t)\|^{2}\right)+t^{2}\left(A^{2} w(t), A w(t)\right)=t\|A w(t)\|^{2}
$$

from which (24) follows after integration and using (23).

The estimates (22)-(24) express in somewhat different ways "parabolic smoothing"; in particular (24) expresses that that the norm of the time derivative $\dot{w}(t)$, or equivalently $A w(t)$, decreases (increases) like $1 / t$ as $t$ increases (decreases), which means that the solution becomes smoother with increasing time. We note a close relation between the two integrals

$$
I_{1}=\int_{0}^{T}\|\dot{w}(t)\| d t=\int_{0}^{T}\|A w(t)\| d t
$$

and

$$
I_{2}=\left(\int_{0}^{T} t\|\dot{w}(t)\|^{2} d t\right)^{1 / 2}=\left(\int_{0}^{T} t\|A w(t)\|^{2} d t\right)^{1 / 2}
$$

both measuring strong stability of (21), with $I_{1}$ through Cauchy's inequality being bounded by $I_{2}$ up to a logarithm:

$$
\int_{\epsilon}^{T}\|A w(t)\| d t \leq\left(\int_{\epsilon}^{T} \frac{1}{t} d t\right)^{1 / 2}\left(\int_{\epsilon}^{T} t\|A w(t)\|^{2} d t\right)^{1 / 2}=(\log (T / \epsilon))^{1 / 2} I_{2} .
$$

Remark 7.1. We now give an argument indicating that for the parabolic model problem (21), the stability factor $S_{c}(T, \psi)$ varies little with the specific choice of data $\psi$. We do this by noting that the quantity $S\left(w^{0}\right)$ defined by

$$
S\left(w^{0}\right)=\left(\int_{0}^{T} t\|A w(t)\|^{2} d t\right)^{1 / 2}
$$

where $w(t)$ solves (21), varies little with the choice of initial data $w^{0}$. To see this, we let $\left\{\chi_{i}\right\}$ be an orthonormal basis for $H$ consisting of eigenfunctions of $A$ with corresponding eigenvalues $\left\{\lambda_{j}\right\}$, which allows us to express the solution $w(t)$ in the form $\sum_{j} \exp \left(-\lambda_{j} t\right) w_{j}^{0} \chi_{j}$ with $w_{j}^{0}=\left(w^{0}, \chi_{j}\right)$. We may then write

$$
\left(S\left(w^{0}\right)\right)^{2}=\int_{0}^{T} t \sum_{j} \lambda_{j}^{2}\left(w_{j}^{0}\right)^{2} \exp \left(-2 \lambda_{j} t\right) d t=\sum_{j}\left(w_{j}^{0}\right)^{2} \int_{0}^{T} t \lambda_{j}^{2} \exp \left(-2 \lambda_{j} t\right) d t
$$

Now, the factor

$$
\int_{0}^{T} t \lambda_{j}^{2} \exp \left(-2 \lambda_{j} t\right) d t=\int_{0}^{T \lambda_{j}} s \exp (-2 s) d s
$$

Encyclopedia of Computational Mechanics. Edited by Erwin Stein, René de Borst and Thomas J.R. Hughes. (C) 2004 John Wiley \& Sons, Ltd. 
takes on almost the same value $\int_{0}^{\infty} s \exp (-2 s) d s \approx 1 / 4$ for all $j$ as soon as $T \lambda_{j} \geq 1$, that is when $\lambda_{j}$ is not very small (since $T$ is typically large). If we randomly choose the initial data $w^{0}$, the chance of hitting an eigenfunction corresponding to a very small eigenvalue, must be very small. We conclude that $S\left(w^{0}\right)$ varies little with $w^{0}$. As just indicated, $S\left(w^{0}\right)$ is related to the integral $\int_{0}^{T}\|\dot{w}(t)\| d t$, which is the analog of the stability factor $S_{c}(T, \psi)$ for the dual problem. The bottom line is that $S_{c}(T, \psi)$ varies little with the choice of $\psi$.

Remark 7.2. The solution operator $\{E(t)\}_{t \geq 0}$ of (21), given by $E(t) w^{0}=w(t)$, is said to define a uniformly bounded and analytic semigroup if there is a constant $S$ such that the following estimates hold:

$$
\begin{aligned}
\|w(t)\| & \leq S\left\|w^{0}\right\|, \\
\|A w(t)\| & \leq \frac{S}{t}\left\|w^{0}\right\|,
\end{aligned}
$$

for $t>0$. We see that that this definition directly couples to the stability estimates of Lemma (7.1), in which case the constant $S$ is of of unit size.

8. Adaptive space-time Galerkin methods for the heat equation

We now move on to space-time Galerkin finite element methods for the model parabolic partial differential equation in the form of the heat equation: Find $u$ : $\Omega \times I \rightarrow \mathbb{R}$ such that

$$
\begin{cases}\dot{u}-\Delta u=f & \text { in } \Omega \times I \\ u=0 & \text { on } \Gamma \times I \\ u(\cdot, 0)=u^{0} & \text { in } \Omega\end{cases}
$$

where $\Omega$ is a bounded domain in $\mathbb{R}^{d}$ with boundary $\Gamma$, on which we have posed homogeneous Dirichlet boundary conditions, $u^{0}$ is a given initial temperature, $f$ a heat source and $I=(0, T]$ a given time interval.

For the discretization of the heat equation in space and time, we use the $c \mathrm{G}(p) \mathrm{dG}(q)$ method based on a tensor product space-time discretization with continuous piecewise polynomial approximation of degree $p \geq 1$ in space and discontinuous piecewise polynomial approximation of degree $q \geq 0$ in time, giving a method which is accurate of order $p+1$ in space and of order $2 q+1$ in time. The discontinuous Galerkin $\operatorname{dG}(q)$ method used for the time discretization reduces to the subdiagonal Padé method for homogeneous constant coefficient problems and in general, together with quadrature for the evaluation of the integral in time, corresponds to an implicit Runge-Kutta method. For the discretization in space we use the standard conforming continuous Galerkin $\mathrm{cG}(p)$ method. The $\mathrm{cG}(p) \mathrm{dG}(q)$ method has maximal flexibility and allows the space and time steps to vary in both space and time. We design and analyze reliable and efficient adaptive algorithms for global error control in $L_{\infty}\left(L_{2}(\Omega)\right.$ ) (maximum in time and $L_{2}$ in space), with possible extensions to $L_{r}\left(L_{s}(\Omega)\right)$ with $1 \leq r, s \leq \infty$. 
The $\mathrm{cG}(p) \mathrm{dG}(q)$ method is based on a partition in time $0=t_{0}<t_{1}<$ $\cdots<t_{N}=T$ of the interval $(0, T]$ into time intervals $I_{n}=\left(t_{n-1}, t_{n}\right]$ of length $k_{n}=t_{n}-t_{n-1}$ with associated finite element spaces $S_{n} \subset H_{0}^{1}(\Omega)$ consisting of piecewise polynomials of degree $p$ on a triangulation $\mathcal{T}_{n}=\{K\}$ of $\Omega$ into elements $K$ with local mesh size given by a function $h_{n}(x)$. We define

$$
V_{n}=\left\{v: v=\sum_{j=0}^{q} t^{j} v_{j}, v_{j} \in S_{n}\right\}
$$

and

$$
V=\left\{v:\left.v\right|_{I_{n}} \in V_{n}, n=1, . ., N\right\} .
$$

We thus define $V$ to be the set of functions $v: \Omega \times I \rightarrow \mathbb{R}$ such that the restriction of $v(x, t)$ to each time interval $I_{n}$ is polynomial in $t$ with coefficients in $S_{n}$. The $\mathrm{cG}(p) \mathrm{dG}(q)$ method for (26) now reads: Find $U \in V$ such that for $n=1,2, \ldots, N$,

$$
\int_{I_{n}}\{(\dot{U}, v)+(\nabla U, \nabla v)\} d t+\left([U]_{n-1}, v_{n-1}^{+}\right)=\int_{I_{n}}(f, v) d t \quad \forall v \in V_{n}
$$

where $[w]_{n}=w\left(t_{n}^{+}\right)-w\left(t_{n}^{-}\right), w_{n}^{+(-)}=\lim _{s \rightarrow 0^{+(-)}} w\left(t_{n}+s\right), U_{0}^{-}=u^{0}$, and $(\cdot, \cdot)$ denotes the $L_{2}(\Omega)$ or $\left[L_{2}(\Omega)\right]^{d}$ inner product. Note that we allow the space discretizations to change with time from one space-time slab $\Omega \times I_{n}$ to the next.

For $q=0$ the scheme (27) reduces to the following variant of the backward Euler scheme:

$$
U_{n}-k_{n} \Delta_{n} U_{n}=P_{n} U_{n-1}+\int_{I_{n}} P_{n} f d t
$$

where $\left.U_{n} \equiv U\right|_{I_{n}}, \Delta_{n}: S_{n} \rightarrow S_{n}$ is the discrete Laplacian on $S_{n}$ defined by $\left(-\Delta_{n} v, w\right)=(\nabla v, \nabla w)$ for all $w \in S_{n}$, and $P_{n}$ is the $L_{2}$-projection onto $S_{n}$ defined by $\left(P_{n} v, w\right)=(v, w)$ for all $w \in S_{n}$.

Alternatively, (28) may be written (with $f \equiv 0$ ) in matrix form as

$$
M_{n} \xi_{n}+k_{n} A_{n} \xi_{n}=M_{n} \hat{\xi}_{n-1},
$$

where $M_{n}$ and $A_{n}$ are mass and stiffness matrices related to a nodal basis for $S_{n}, \xi_{n}$ is the corresponding vector of nodal values for $U_{n}$, and $\hat{\xi}_{n-1}$ is the vector of nodal values for $P_{n} U_{n-1}$. Evidently, we have to solve a system of equations with system matrix $M_{n}+k_{n} A_{n}$ to compute $\xi_{n}$.

Remark 8.1. Note that in the discretization (27), the space and time steps may vary in time and that the space discretization may be variable also in space, whereas the time steps $k_{n}$ are kept constant in space. Clearly, optimal mesh design requires the time steps to be variable also in space. Now, it is easy to extend the method (27) to admit time steps which are variable in space simply by defining

$$
V_{n}=\left\{v:\left.v\right|_{I_{n}}=\sum_{j} c_{j}(t) v_{j}, v_{j} \in S_{n}\right\}
$$


where now the coefficients $c_{j}(t)$ are piecewise polynomial of degree $q$ in $t$ without continuity requirements, on partitions of $I_{n}$ which may vary with $j$. The discrete functions may now be discontinuous in time also inside the space-time slab $\Omega \times I_{n}$, and the degree $q$ may vary over components and subintervals. The $\mathrm{cG}(p) \mathrm{dG}(q)$ method again takes the form (27), with the term $\left([U]_{n-1}, v_{n-1}^{+}\right)$replaced by a sum over all jumps in time of $U$ in $\Omega \times\left[t_{n-1}, t_{n}\right)$. Adaptive methods in this generality, so-called multi-adaptive methods, are proposed and analyzed in detail for systems of ordinary differential equations in (Logg, 2001a, Logg, 2001b).

\section{A priori and a posteriori error estimates for the heat equation}

In this section we state a priori and a posteriori error estimates for the $c \mathrm{G}(p) \mathrm{dG}(q)$ method (27) in the case $p=1$ and $q=0,1$, and give the proofs below. A couple of technical assumptions on the space-mesh function $h_{n}(x)$ and time steps $k_{n}$ are needed: We assume that each triangulation $\mathcal{T}_{n}$ with associated mesh size $h_{n}$ satisfies, with $h_{n, K}$ equal to the diameter and $m_{n, K}$ the volume of $K \in \mathcal{T}_{n}$,

$$
\begin{gathered}
c_{1} h_{n, K}^{d} \leq m_{n, K} \quad \forall K \in \mathcal{T}_{n}, \\
c_{2} h_{n, K} \leq h_{n}(x) \leq h_{n, K} \quad \forall x \in K \quad \forall K \in T_{h}, \\
\left|\nabla h_{n}(x)\right| \leq \mu \quad \forall x \in \Omega,
\end{gathered}
$$

for some positive constants $c_{1}, c_{2}$ and $\mu$. The constant $\mu$ will be assumed to be small enough in the a priori error estimates (but not in the a posteriori error estimates). We further assume that there are positive constants $c_{3}, c_{4}$ and $\gamma$ such that for all $n$ we have

$$
\begin{gathered}
k_{n} \leq c_{3} k_{n+1}, \\
c_{4} h_{n}(x) \leq h_{n+1}(x) \leq \frac{1}{c_{4}} h_{n}(x) \quad \forall x \in \Omega, \\
\bar{h}_{n}^{2} \leq \gamma k_{n} \quad \text { or } \quad S_{n} \subset S_{n-1},
\end{gathered}
$$

where $\bar{h}_{n}=\max _{x \in \bar{\Omega}} h_{n}(x)$. Furthermore, we assume for simplicity that $\Omega$ is convex, so that the following elliptic regularity estimate holds: $\left\|D^{2} v\right\| \leq\|\Delta v\|$ for all functions $v$ vanishing on $\Gamma$. Here $\left(D^{2} v\right)^{2}=\sum_{i j} v_{, i j}^{2}$, where $v_{, i j}$ is the second partial derivative of $v$ with respect to $x_{i}$ and $x_{j}$, and $\|\cdot\|$ denotes the $L_{2}(\Omega)$-norm. With these assumptions, we have the following a priori error estimates:

Theorem 9.1. If $\mu$ and $\gamma$ are sufficiently small, then there is a constant $C$ only depending on the constants $c_{i}, i=1,2,3,4$, such that for $u$ the solution of (26) and $U$ that of (27), we have for $p=1$ and $q=0,1$,

$$
\|u-U\|_{I_{n}} \leq C L_{n} \max _{1 \leq m \leq n} E_{q m}(u), \quad n=1, \ldots, N,
$$

and for $q=1$,

$$
\left\|u\left(t_{n}\right)-U\left(t_{n}\right)\right\| \leq C L_{n} \max _{1 \leq m \leq n} E_{2 m}(u), \quad n=1, \ldots, N,
$$


where $L_{n}=\left(\log \left(t_{n} / k_{n}\right)+1\right)^{1 / 2}, E_{q m}(u)=\min _{j \leq q+1} k_{m}^{j}\left\|u_{t}^{(j)}\right\|_{I_{m}}+\left\|h_{m}^{2} D^{2} u\right\|_{I_{m}}$, $q=0,1,2$ with $u^{(1)}=\dot{u}, u_{t}^{(2)}=\ddot{u}, u_{t}^{(3)}=\Delta \ddot{u}$ and $\|w\|_{I_{m}}=\max _{t \in I_{m}}\|w(t)\|$.

These estimates state that the discontinuous Galerkin method (27) is of order $q+1$ globally in time and of order $2 q+1$ at the discrete time levels $t_{n}$ for $q=0,1$, and is second order in space. In particular, the estimate (35) is optimal compared to interpolation with piecewise polynomials of order $q=0,1$ in time and piecewise linears in space, up to the logarithmic factor $L_{n}$. The third order accuracy in time at the discrete time levels for $q=1$ reflects a superconvergence feature of the $\mathrm{dG}(q)$ method.

The a posteriori error estimates for (27) take the form:

Theorem 9.2. If $u$ is the solution of (26) and $U$ that of (27) with $p=1$, then we have for $q=0$,

$$
\left\|u\left(t_{n}\right)-U\left(t_{n}\right)\right\| \leq \max _{1 \leq m \leq n} \mathcal{E}_{0 m}(U), \quad n=1, \ldots, N
$$

and for $q=1$,

$$
\left\|u\left(t_{n}\right)-U\left(t_{n}\right)\right\| \leq \max _{1 \leq m \leq n} \mathcal{E}_{2 m}(U), \quad n=1, \ldots, N
$$

where

$$
\begin{aligned}
& \mathcal{E}_{0 m}(U)=\gamma_{1}\left\|h_{m}^{2} R(U)\right\|_{I_{m}}+\gamma_{2}\left\|h_{m}^{2}[U]_{m-1} / k_{m}\right\|^{*}+\gamma_{3}\left\|k_{m} R_{0 k}(U)\right\|_{I_{m}}, \\
& \mathcal{E}_{2 m}(U)=\gamma_{1}\left\|h_{m}^{2} R(U)\right\|_{I_{m}}+\gamma_{2}\left\|h_{m}^{2}[U]_{m-1} / k_{m}\right\|^{*}+\gamma_{4}\left\|k_{m}^{3} R_{1 k}(U)\right\|_{I_{m}}
\end{aligned}
$$

and

$$
\begin{aligned}
R(U) & =|f|+D_{m}^{2} U \\
R_{0 k}(U) & =|f|+\left|[U]_{m-1}\right| / k_{m} \\
R_{1 k}(U) & =\left|f_{t t}\right|+\left|\Delta_{m} P_{m}[U]_{m-1}\right| / k_{m}^{2}
\end{aligned}
$$

on $\Omega \times I_{m}$. A star indicates that the corresponding term is present only if $S_{m-1}$ is not a subset of $S_{m}$. Further, $\gamma_{i}=L_{N} C_{i}$ where the $C_{i}$ are constants related to approximation by piecewise constant or linear functions. Finally, $D_{m}^{2} U$ on a space element $K \in \mathcal{T}_{m}$ is the modulus of the maximal jump in normal derivative of $U$ across an edge of $K$ divided by the diameter of $K$.

Remark 9.1. The term $|f|$ in $R(U)$ may a be replaced by $\left|h_{m}^{2} D^{2} f\right|_{\text {.: Similarly, the }}$ term $|f|$ in $R_{0 k}$ may be replaced with $k|\dot{f}|$ and $|\ddot{f}|$ in $R_{1 k}(U)$ by $k|\Delta \ddot{f}|$.

The a posteriori error estimates are sharp in the sense that the quantities on the righthand sides can be bounded by the corresponding right-hand sides in the (optimal) a priori error estimates. Therefore, the a posteriori error estimates may be used as a basis for efficient adaptive algorithms, as we indicate below. 
10. Adaptive methods/algorithms

An adaptive method for the heat equation addresses the following problem: For a given tolerance TOL $>0$ find a discretization in space and time $\mathcal{S}_{h k}=\left\{\left(\mathcal{T}_{n}, k_{n}\right)\right\}_{n \geq 1}$, such that

(1) $\left\|u\left(t_{n}\right)-U\left(t_{n}\right)\right\| \leq$ TOL for $n=1,2, \ldots$,

(2) $\mathcal{S}_{h k}$ is optimal, in the sense that the number of degrees of freedom is minimal.

We approach this problem using the a posteriori estimates (37) and (38) in an adaptive method of the form: Find $\mathcal{S}_{h k}$ such that for $n=1,2, \ldots$,

$$
\begin{aligned}
& \mathcal{E}_{o n}(U) \leq \mathrm{TOL}, \quad \text { if } q=0, \\
& \mathcal{E}_{2 n}(U) \leq \mathrm{TOL}, \quad \text { if } q=1,
\end{aligned}
$$

the number of degrees of freedom of $\mathcal{S}_{h k}$ is minimal.

To solve this problem, we use an adaptive algorithm for choosing $\mathcal{S}_{h k}$ based on equidistribution of the form: For each $n=1,2, \ldots$, with $\mathcal{T}_{n 0}$ a given initial space mesh and $k_{n 0}$ an initial time step, determine triangulations $\mathcal{T}_{n j}$ with $N_{j}$ elements of size $h_{n j}(x)$, time steps $k_{n j}$, and corresponding approximate solutions $U_{n j}$ defined on $I_{n j}=\left(t_{n-1}, t_{n-1}+k_{n j}\right)$, such that for $j=0,1, \ldots, \hat{n}-1$,

$$
\begin{aligned}
& \gamma_{1} \max _{t \in I_{n j}}\left\|h_{n, j+1}^{2} R\left(U_{n j}\right)\right\|_{L_{2}(K)} \\
& +\gamma_{2}\left\|h_{n, j+1}^{2}[U]_{n-1, j} / k_{n j}\right\|_{L_{2}(K)}^{*}=\frac{\theta \mathrm{TOL}}{2 \sqrt{N_{j}}} \forall K \in T_{n j}, \\
& k_{n, j+1} \gamma_{3}\left\|R_{0 k}\left(U_{n j}\right)\right\|_{I_{n j}}=\frac{\mathrm{TOL}}{2}, \quad \text { if } q=0, \\
& k_{n, j+1}^{3} \gamma_{4}\left\|R_{1 k}\left(U_{n j}\right)\right\|_{I_{n j}}=\frac{\mathrm{TOL}}{2}, \quad \text { if } q=1,
\end{aligned}
$$

that is we determine iteratively each new time step $k_{n}=k_{n \hat{n}}$ and triangulation $\mathcal{T}_{n}=\mathcal{T}_{n \hat{n}}$. The number of trials $\hat{n}$ is the smallest integer $j$ such that (40) holds with $U$ replaced by $U_{n j}$, and the parameter $\theta \sim 1$ is chosen so that $\hat{n}$ is small.

\section{Reliability and efficiency.}

By the a posteriori estimates (37) and (38) it follows that the adaptive method (40) is reliable in the sense that if (40) holds, then the error control (39) is guaranteed. The efficiency of (40) follows from the fact that the right-hand sides of the a posteriori error estimates may be bounded by the corresponding right-hand sides in the (optimal) a priori error estimates. 
12. Strong stability estimates for the heat equation

We now state the fundamental strong stability results for the continuous and discrete problems to be used in the proofs of the a priori and a posteriori error estimates. Analogous to in Section 7, we consider the problem $\dot{w}-\Delta w=0$, where $w(t)=$ $\phi(T-t)$ is the backward dual solution with time reversed.

The proof of Lemma 12.1 is similar to that of Lemma 7.1, multiplying by $w(t)$, $-t \Delta w(t)$ and $t^{2} \Delta^{2} w(t)$. The proof of Lemma 12.2 is also analogous: For $q=0$, we multiply (28) by $W_{n}$ and $t_{n} A_{n} W_{n}$, noting that if $S_{n-1} \subset S_{n}$ (corresponding to coarsening in the time direction of the primal problem $\dot{u}-\Delta u=f$ ), then $P_{n} W_{n-1}=W_{n-1},\left(A_{n} W_{n}, W_{n-1}\right)=\left(W_{n}, A_{n} W_{n-1}\right)$ and $\left(A_{n} W_{n-1}, W_{n-1}\right)=$ $\left(A_{n-1} W_{n-1}, W_{n-1}\right)$. The proof for $q=1 \mathrm{i}$ similar.

Lemma 12.1. Let $w$ be the solution of (26) with $f \equiv 0$. Then for $T>0$,

$$
\begin{gathered}
\|w(T)\|^{2}+2 \int_{0}^{T}\|\nabla w(t)\|^{2} d t=\left\|w^{0}\right\|^{2}, \\
\int_{0}^{T} T\left\{\|\dot{w}(t)\|^{2}+\|\Delta w(t)\|^{2}\right\} d t \leq \frac{1}{2}\left\|w^{0}\right\|^{2}, \\
\|\Delta w(T)\| \leq \frac{1}{\sqrt{2} T}\left\|w^{0}\right\| .
\end{gathered}
$$

Lemma 12.2. There is a constant $C$ such that if $S_{n-1} \subset S_{n}$ for $n=1,2, \ldots, N$, and $W$ is the solution of (27) with $f \equiv 0$, then for $T=t_{N}>0$,

$$
\begin{gathered}
\left\|W_{N}^{-}\right\|^{2}+2 \int_{0}^{T}\|\nabla W\|^{2} d t+\sum_{n=1}^{N}\left\|[W]_{n-1}\right\|^{2}=\left\|w^{0}\right\|^{2} \\
\sum_{n=1}^{N} t_{n} \int_{I_{n}}\left\{\|\dot{W}\|^{2}+\left\|\Delta_{n} W\right\|^{2}\right\} d t+\sum_{n=1}^{N} t_{n}\left\|[W]_{n-1}\right\|^{2} / k_{n} \leq C\left\|w^{0}\right\|^{2},
\end{gathered}
$$

and

$$
\sum_{n=1}^{N} \int_{I_{n}}\left\{\|\dot{W}\|+\left\|\Delta_{n} W\right\|\right\} d t+\sum_{n=1}^{N}\left\|[W]_{n-1}\right\| \leq C\left(\log \frac{t_{n}}{k_{1}}+1\right)^{1 / 2}\left\|w^{0}\right\| .
$$

13. A priori error estimates for the $L_{2}$ - and elliptic projections

We shall use the following a priori error estimate for the $L_{2}$-projection $P_{n}: L_{2}(\Omega) \rightarrow$ $S_{n}$ defined by $\left(w-P_{n} w, v\right)=0$ for all $v \in S_{n}$. This estimate follows from the fact that $P_{n}$ is very close to the nodal interpolation operator $J_{n}$ into $S_{n}$, defined by $J_{n} w=w$ at the nodes of $\mathcal{T}_{n}$ if $w$ is smooth (and $J_{n} w=J_{n} \tilde{w}$ if $w \in H^{1}(\Omega)$, where $\tilde{w}$ is a locally regularized approximation of $w$ ).

Encyclopedia of Computational Mechanics. Edited by Erwin Stein, René de Borst and Thomas J.R. Hughes. (c) 2004 John Wiley \& Sons, Ltd. 
Lemma 13.1. If $\mu$ is sufficiently small, then there is a positive constant $C$ such that for all $w \in H_{0}^{1}(\Omega) \cap H^{2}(\Omega)$,

$$
\left|\left(f, w-P_{n} w\right)-\left(\nabla U, \nabla\left(w-P_{n} w\right)\right)\right| \leq C\left\|h_{n}^{2} R_{n}(U)\right\|\left\|D^{2} w\right\|,
$$

where $R_{n}(U)=|f|+D_{n}^{2} U$.

We shall also need the following a priori error estimate for the elliptic projection $\pi_{n}: H_{0}^{1}(\Omega) \rightarrow S_{n}$ defined by

$$
\left(\nabla\left(w-\pi_{n} w\right), \nabla v\right)=0 \quad \forall v \in S_{n} .
$$

Lemma 13.2. If $\mu$ is sufficiently small, then there is a positive constant $C$ such that for all $w \in H^{2}(\Omega) \cap H_{0}^{1}(\Omega)$,

$$
\left\|w-\pi_{n} w\right\| \leq C\left\|h_{n}^{2} D^{2} w\right\| .
$$

Proof. We shall first prove that with $e=w-\pi_{n} w$, we have $\|e\| \leq C\left\|h_{n} \nabla e\right\|$. For this purpose, we let $\phi$ be the solution of the continuous dual problem $-\Delta \phi=e$ in $\Omega$ with $\phi=0$ on $\Gamma$, and note that by integration by parts, the Galerkin orthogonality (49), a standard estimate for the interpolation error $u-J_{n} u$, together with elliptic regularity, we have

$$
\begin{aligned}
\|e\|^{2} & =(e,-\Delta \phi)=(\nabla e, \nabla \phi)=\left(\nabla e, \nabla\left(\phi-J_{n} \phi\right)\right) \\
& \leq\left\|h_{n} \nabla e\right\|\left\|h_{n}^{-1} \nabla\left(\phi-J_{n} \phi\right)\right\| \leq C\left\|h_{n} \nabla e\right\|\left\|D^{2} \phi\right\| \leq C\left\|h_{n} \nabla e\right\|\|e\|,
\end{aligned}
$$

which proves the desired estimate. Next to prove that $\left\|h_{n} \nabla e\right\| \leq C\left\|h_{n}^{2} D^{2} w\right\|$, we note that since $\pi_{n} J_{n} u=J_{n} u$, we have

$$
\begin{aligned}
\left\|h_{n} \nabla e\right\| & \leq\left\|h_{n} \nabla\left(w-J_{n} w\right)\right\|+\left\|h_{n} \nabla \pi_{n}\left(w-J_{n} w\right)\right\| \\
& \leq C\left\|h_{n} \nabla\left(w-J_{n} w\right)\right\| \leq C\left\|h_{n}^{2} D^{2} w\right\|_{2},
\end{aligned}
$$

where we used stability of the elliptic projection $\pi_{n}$ in the form

$$
\left\|h_{n} \nabla \pi_{n} v\right\| \leq C\left\|h_{n} \nabla v\right\| \quad \forall v \in H_{0}^{1}(\Omega),
$$

which is a weighted analog of the basic property of the elliptic projection $\left\|\nabla \pi_{n} v\right\| \leq$ $\|\nabla v\|$ for all $v \in H_{0}^{1}(\Omega)$. For the proof of the weighted analog we need the mesh size not to vary too quickly, expressed in the assumption that $\mu$ is small.

\section{Proof of the a priori error estimates}

In this section we give the proof of the a priori estimates, including (35) and (36). For simplicity, we shall assume that $S_{n} \subset S_{n-1}$, corresponding to a situation where the solution gets smoother with increasing time. The proof is naturally divided into the following steps, indicating the overall structure of the argument:

(a) An error representation formula using duality;

(b) Strong stability of the discrete dual problem;

(c) Choice of interpolant and proof of (35);

(d) Choice of interpolant and proof of (36). 


\subsection{An error representation formula using duality}

Given a discrete time level $t_{N}>0$, we write the discrete set of equations (27) determining the discrete solution $U \in V$ up to time $t_{N}$ in compact form as

$$
A(U, v)=\left(u^{0}, v_{0}^{+}\right)+(f, v)_{I} \quad \forall v \in V,
$$

where

$$
A(w, v) \equiv \sum_{n=1}^{N}\left\{(\dot{w}, v)_{n}+(\nabla w, \nabla v)_{n}\right\}+\left(w_{0}^{+}, v_{0}^{+}\right)+\sum_{n=2}^{N}\left([w]_{n-1}, v_{n-1}^{+}\right),
$$

$(v, w)_{n}=\int_{I_{n}}(v, w) d t$ and $I=(0, T]$. The error $e \equiv u-U$ satisfies the Galerkin orthogonality

$$
A(e, v)=0 \quad \forall v \in V,
$$

which follows from the fact that (51) is satisfied also by the exact solution $u$ of (26). Let now the discrete dual solution $\Phi \in V$ be defined by

$$
A(v, \Phi)=\left(v_{N}^{-}, e_{N}\right) \quad \forall v \in V
$$

where $e_{N}=u\left(t_{N}\right)-U\left(t_{N}\right)$ is the error at final time $t_{N}$, corresponding to control of the $L_{2}(\Omega)$-norm of $e_{N}$. We note that $\Phi$ is a discrete $\mathrm{cG}(p) \mathrm{dG}(q)$-solution of the continuous dual problem

$$
\begin{aligned}
-\dot{\phi}-\Delta \phi & =0 \quad \text { in } \Omega \times[0, T), \\
\phi & =0 \quad \text { on } \Gamma \times[0, T),
\end{aligned}
$$

with initial data $\phi(T)=e_{N}$. This follows from the fact that the bilinear form $A(\cdot, \cdot)$, after time integration by parts, can also be written as

$$
A(w, v)=\sum_{n=1}^{N}\left\{(w,-\dot{v})_{n}+(\nabla w, \nabla v)_{n}\right\}+\sum_{n=1}^{N-1}\left(w_{n}^{-},-[v]_{n}\right)+\left(w_{N}^{-}, v_{N}^{-}\right)
$$

In view of (53) and (52) we have for any $v \in V$,

$$
\begin{aligned}
\left\|e_{N}\right\|^{2} & =\left(u_{N}-v_{N}^{-}, e_{N}\right)+\left(v_{N}^{-}-U_{N}^{-}, e_{N}\right) \\
& =\left(u_{N}-v_{N}^{-}, e_{N}\right)+A(v-U, \Phi)=\left(u_{N}-v_{N}^{-}, e_{N}\right)+A(v-u, \Phi) .
\end{aligned}
$$

Taking here $v \in V$ to be a suitable interpolant of $u$, we thus obtain a representation of the error $e_{N}$ in terms of an interpolation error $u-v$ and the discrete solution $\Phi$ of the associated dual problem, combined through the bilinear form $A(\cdot, \cdot)$. To obtain the a priori error estimates, we estimate below the interpolation error $u-v$ in $L_{\infty}\left(L_{2}(\Omega)\right)$ and the time derivative $\dot{\Phi}$ in $L_{1}\left(L_{2}(\Omega)\right)$ using discrete strong stability. 


\subsection{Strong stability of the discrete dual problem}

We apply Lemma 12.2 to the function $w(t)=\Phi(T-t)$, to obtain the strong stability estimate

$$
\|\Phi(t)\|_{I}+\sum_{n=1}^{N} \int_{I_{n}}\left\{\|\dot{\Phi}\|+\left\|\Delta_{n} \Phi\right\|\right\} d t+\sum_{n=1}^{N}\left\|[\Phi]_{n}\right\| \leq C L_{N}\left\|e_{N}\right\|,
$$

with $L_{N}=\left(\log \left(T_{N} / k_{n}\right)+1\right)^{1 / 2}$.

\subsection{Proof of the a priori error estimate (35)}

In the error representation we take the interpolant to be $v=\tilde{u} \equiv Q_{n} \pi_{n} u$ on $I_{n}$, where $Q_{n}$ is the $L_{2}\left(I_{n}\right)$-projection onto polynomials of degree $q$ on $I_{n}$, and $\pi_{n}$ is the elliptic projection defined in Section 13. For $q=0$, we thus take

$$
\left.\tilde{u}\right|_{I_{n}}=k_{n}^{-1} \int_{I_{n}} \pi_{n} u d s,
$$

and for $q=1$, we take

$$
\left.\tilde{u}\right|_{I_{n}}=k_{n}^{-1} \int_{I_{n}} \pi_{n} u d s+\frac{12\left(t-t_{n-1}-k_{n} / 2\right)}{k_{n}^{3}} \int_{I_{n}}\left(s-t_{n-1}-k_{n} / 2\right) \pi_{n} u d s .
$$

With this choice of interpolant, (56) reduces to

$$
\begin{aligned}
\left\|e_{N}\right\|^{2}= & \left(u_{N}-\tilde{u}_{N}^{-}, e_{N}\right)+\sum_{n=1}^{N}\left(u-\pi_{n} u, \dot{\Phi}\right)_{n} \\
& +\sum_{n=1}^{N-1}\left(u_{n}-\tilde{u}_{n}^{-},[\Phi]_{n}\right)-\left(u_{N}-\tilde{u}_{N}^{-}, \Phi_{N}^{-}\right),
\end{aligned}
$$

where we have used (49), (55) and the fact that $\left(\pi_{n} u-\tilde{u}, v\right)_{n}=0$ for all $v \in V_{n}$, and thus in particular for $v=\dot{\Phi}$ and $v=\Delta_{n} \Phi$.

Using Lemma 13.2 and the fact that $Q_{n}$ is bounded in $\|\cdot\|_{I_{n}}$, we have

$$
\begin{aligned}
\|u-\tilde{u}\|_{I_{n}} & \leq\left\|u-Q_{n} u\right\|_{I_{n}}+\left\|Q_{n}\left(u-\pi_{n} u\right)\right\|_{I_{n}} \\
& \leq C\left(\min _{j \leq q+1} k_{n}^{j}\left\|u_{t}^{(j)}\right\|_{I_{n}}+\left\|h_{n}^{2} D^{2} u\right\|_{I_{n}}\right),
\end{aligned}
$$

where the bound for $u-Q_{n} u$ follows from the Taylor expansion

$$
\begin{aligned}
u(t) & =u\left(t_{n}\right)+\int_{t_{n}}^{t} \dot{u}(s) d s \\
& =u\left(t_{n}\right)+\left(t-t_{n}\right)+\dot{u}\left(t_{n}\right)+\int_{t_{n}}^{t}(t-s) \ddot{u}(s) d s,
\end{aligned}
$$

noting that $Q_{n}$ is the identity on the polynomial part of $u(t)$. 
From (60) we thus obtain,

$$
\begin{gathered}
\left\|e_{N}\right\|^{2} \leq C \max _{1 \leq n \leq N}\left(\min _{j \leq q+1} k_{n}^{j}\left\|u_{t}^{(j)}\right\| I_{I_{n}}+\left\|h_{n}^{2} D^{2} u\right\|_{I_{n}}\right) \\
\times\left(\left\|e_{N}\right\|+\sum_{n=1}^{N} \int_{I_{n}}\|\dot{\Phi}\| d t+\sum_{n=1}^{N-1}\left\|[\Phi]_{n}\right\|+\left\|\Phi_{N}^{-}\right\|\right),
\end{gathered}
$$

and conclude in view of (57) that

$$
\left\|e_{N}\right\| \leq C L_{N} \max _{1 \leq n \leq N}\left(\min _{j \leq r+1} k_{n}^{j}\left\|u_{t}^{(j)}\right\|_{I_{n}}+\left\|h_{n}^{2} D^{2} u\right\|_{I_{n}}\right) .
$$

By a local analysis this estimate extends to $\|e\|_{I_{N}}$, completing the proof of (35).

\subsection{Proof of the a priori error estimate (36)}

In the error representation formula (56) we now choose $v=R_{n} \pi_{n} u$, where $R_{n}$ is the (Radau) projection onto linear functions on $I_{n}$, defined by $\left(R_{n} \pi_{n} u\right)_{n}^{-}=\pi_{n} u_{n}$ and the condition that $R_{n} \pi_{n} u-\pi_{n} u$ has mean value zero over $I_{n}$, that is we take

$$
\left.R_{n} \pi_{n} u\right|_{I_{n}}=\pi_{n} u_{n}+\left(t-t_{n}\right) \frac{2}{k_{n}^{2}} \int_{I_{n}} \pi_{n}\left(u_{n}-u\right) d s .
$$

With this choice of interpolant, (56) reduces to

$$
\begin{aligned}
\left\|e_{N}\right\|^{2}= & \left(u_{N}-\pi_{N} u_{N}, e_{N}\right) \\
& +\sum_{n=1}^{N}\left(u-\pi_{n} u, \dot{\Phi}\right)_{n}-\sum_{n=1}^{N}\left(\nabla\left(\pi_{n} u-R_{n} \pi_{n} u\right), \nabla \Phi\right)_{n} \\
& +\sum_{n=1}^{N-1}\left(u_{n}-\pi_{n} u_{n},[\Phi]_{n}\right)-\left(u_{N}-\pi_{N} u_{N}, \Phi_{N}^{-}\right),
\end{aligned}
$$

where in the first sum we have used the fact that $\pi_{n} u-R_{n} \pi_{n} u$ is orthogonal to $\dot{\Phi}$ (which is constant in $t$ on $I_{n}$ ), and in the second sum we have used (49). For the latter term, we have

$$
\begin{aligned}
\left(\nabla\left(\pi_{n} u-R_{n} \pi_{n} u\right), \nabla \Phi\right)_{n}= & \left(\nabla\left(\pi_{n} u-R_{n} \pi_{n} u\right), \nabla \Phi_{n}^{-}\right)_{n} \\
& +\left(\nabla\left(\pi_{n} u-R_{n} \pi_{n} u\right),\left(t-t_{n}\right) \nabla \dot{\Phi}\right)_{n}
\end{aligned}
$$

so that by our choice of $R_{n} \pi_{n} u$,

$$
\begin{aligned}
\left|\left(\nabla\left(\pi_{n} u-R_{n} \pi_{n} u\right), \nabla \Phi\right)_{n}\right| & =\left|\left(\nabla\left(\pi_{n} u-R_{n} \pi_{n} u\right),\left(t-t_{n}\right) \nabla \dot{\Phi}\right)_{n}\right| \\
& \leq k_{n}\left\|\Delta_{n}\left(\pi_{n} u-R_{n} \pi_{n} u\right)\right\|_{I_{n}} \int_{I_{n}}\|\dot{\Phi}\| d t
\end{aligned}
$$

Using Taylor expansions we easily find that

$$
\left\|\Delta_{n}\left(\pi_{n} u-R_{n} \pi_{n} u\right)\right\|_{I_{n}} \leq C k_{n}^{2}\left\|\Delta_{n} \pi_{n} u_{t}^{(2)}\right\|_{I_{n}} .
$$

Finally, we note that for any $w \in H^{2}(\Omega) \cap H_{0}^{1}(\Omega)$ we have

$$
\left(-\Delta_{n} \pi_{n} w, v\right)=\left(\nabla \pi_{n} w, \nabla v\right)=(\nabla w, \nabla v)=(-\Delta w, v) \quad \forall v \in S_{n}
$$


from which we deduce by taking $v=-\Delta_{n} \pi_{n} w$ that

$$
\left\|\Delta_{n} \pi_{n} w\right\| \leq\|\Delta w\| \quad \forall w \in H^{2}(\Omega) \cap H_{0}^{1}(\Omega) .
$$

It now follows from (64) through (67), together with Lemma 13.2 and strong stability for $\Phi$, that

$$
\begin{aligned}
\left\|e_{N}\right\|^{2} \leq & C \max _{1 \leq n \leq N}\left(\min _{j \leq q+2} k_{n}^{j}\left\|u_{t}^{(j)}\right\|_{I_{n}}+\left\|h_{n}^{2} D^{2} u\right\|_{I_{n}}\right) \\
& \times\left(\left\|e_{N}\right\|+\sum_{n=1}^{N} \int_{I_{n}}\|\dot{\Phi}\| d t+\sum_{n=1}^{N-1}\left\|[\Phi]_{n}\right\|+\left\|\Phi_{N}^{-}\right\|\right) \\
\leq & \left\|e_{N}\right\| C L_{N} \max _{1 \leq n \leq N}\left(\min _{j \leq q+2} k_{n}^{j}\left\|u_{t}^{(j)}\right\|_{I_{n}}+\left\|h_{n}^{2} D^{2} u\right\|_{I_{n}}\right),
\end{aligned}
$$

where we have used the notation $u_{t}^{(3)}=\Delta \ddot{u}$. This completes the proof of (36) and Theorem 9.1.

\section{Proof of the a posteriori error estimates}

The proof of the a posteriori error estimates is similar to that of the a priori error estimates just presented. The difference is that now the error representation involves the exact solution $\phi$ of the continuous dual problem (54), together with the residual of the discrete solution $U$. By the definition of the dual problem and the bilinear form $A$, we have

$$
\left\|e_{N}\right\|^{2}=A(e, \phi)=A(u, \phi)-A(U, \phi)
$$

Using now that

$$
A(u, \phi)=\left(u^{0}, \phi_{0}^{+}\right)+(f, \phi)_{I}
$$

together with the Galerkin orthogonality, we obtain the following error representation in terms of the discrete solution $U$, the dual solution $\phi$ and data $u^{0}$ and $f$ :

$$
\begin{aligned}
\left\|e_{N}\right\|^{2}= & A(U, v-\phi)+\left(u^{0}, \phi_{0}^{+}-v_{0}^{+}\right)+(f, \phi-v)_{I} \\
= & \sum_{n=1}^{N}\left\{(\dot{U}, v-\phi)_{n}+(\nabla U, \nabla(v-\phi))_{n}\right\}+\sum_{n=1}^{N}\left([U]_{n-1}, v_{n-1}^{+}-\phi_{n-1}^{+}\right) \\
& +(f, \phi-v)_{I} \\
= & I+I I+I I I
\end{aligned}
$$

with obvious notation. This holds for all $v \in V$.

To prove (37), we now choose $\left.v\right|_{I_{n}}=\tilde{\phi} \equiv Q_{n} P_{n} \phi$ in (68), and note that

$$
(\dot{U}, \tilde{\phi}-\phi)_{n}=0 \text {. }
$$

Since also

$$
\left(\nabla U, \nabla\left(\tilde{\phi}-P_{n} \phi\right)\right)_{n}=\left(-\Delta_{n} U, \tilde{\phi}-P_{n} \phi\right)_{n}=\left(-\Delta_{n} U,\left(Q_{n}-I\right) P_{n} \phi\right)=0
$$


it follows that

$$
I=\sum_{n=1}^{N}\left(\nabla U, \nabla\left(P_{n}-I\right) \phi\right)_{n}=\sum_{n=1}^{N}\left(\nabla U_{n}, \nabla\left(P_{n}-I\right) \int_{I_{n}} \phi d t\right) .
$$

Using that

$$
\Delta \int_{I_{n}} \phi d t=\int_{I_{n}} \Delta \phi d t=\int_{I_{n}} \dot{\phi} d t=\phi\left(t_{n}\right)-\phi\left(t_{n-1}\right),
$$

together with Lemma 13.1 and elliptic regularity, we get

$$
\begin{aligned}
|I| & \leq C \sum_{n=1}^{N}\left\|h_{n}^{2} D_{n}^{2} U\right\|_{I_{n}}\left\|\Delta \int_{I_{n}} \phi d t\right\| \\
& \leq C \max _{1 \leq n \leq N}\left\|h_{n}^{2} D_{n}^{2}(U)\right\|_{I_{n}}\left(\int_{0}^{t_{N-1}}\|\dot{\phi}\| d t+2\|\phi\|_{I_{N}}\right) .
\end{aligned}
$$

To estimate $I I$, we note that by Lemma 13.1 we have

$$
\left|\left([U]_{n-1},\left(P_{n}-I\right) \phi_{n-1}^{+}\right)\right| \leq C\left\|h_{n}^{2}[U]_{n-1}\right\|^{*}\left\|\Delta \phi_{n-1}^{+}\right\|,
$$

noting that the left-hand side is zero if $S_{n-1} \subset S_{n}$. By obvious stability and approximation properties of the $L_{2}\left(I_{n}\right)$-projections onto the set of constant functions on $I_{n}$, we also have

$$
\left\|\tilde{\phi}-P_{n} \phi\right\|_{I_{n}} \leq\left\|P_{n} \phi\right\|_{I_{n}} \leq\|\phi\|_{I_{n}}
$$

and

$$
\left\|\tilde{\phi}-P_{n} \phi\right\|_{I_{n}} \leq \int_{I_{n}}\left\|P_{n} \dot{\phi}\right\| d t \leq \int_{I_{n}}\|\dot{\phi}\| d t .
$$

We thus conclude that

$$
\begin{aligned}
|I I| \leq & C \max _{1 \leq n \leq N}\left\|h_{n}^{2}[U]_{n-1} / k_{n}\right\|^{*} \sum_{n=1}^{N} k_{n}\left\|\Delta \phi_{n-1}^{+}\right\| \\
& +\max _{1 \leq n \leq N}\left\|[U]_{n-1}\right\|\left(\int_{o}^{t_{N-1}}\|\dot{\phi}\| d t+\|\phi\|_{I_{N}}\right) .
\end{aligned}
$$

The data term III is estimated similarly. We finally use strong stability of $\phi$ in the form

$$
\begin{aligned}
\sum_{n=1}^{N-1} k_{n}\left\|w_{n-1}^{+}\right\| & \leq \int_{0}^{t_{N-1}}\|w\| d t \\
& \leq\left(\int_{0}^{t_{N-1}}\left(t_{N}-t\right)^{-1} d t\right)^{1 / 2}\left(\int_{0}^{t_{N}}\left(t_{N}-t\right)\|w\|^{2} d t\right)^{1 / 2} \\
& \leq \frac{1}{2}\left(\log \frac{t_{N}}{k_{N}}\right)^{1 / 2}\left\|e_{N}\right\|,
\end{aligned}
$$

for $w=\dot{\phi}$ and $w=\Delta \phi$, together with the estimate $k_{N}\left\|\Delta \phi_{N-1}^{+}\right\| \leq \exp (-1)\left\|e_{N}\right\|$.

Combining the estimates completes the proof of the posteriori error estimate (37). The proof of the a posteriori error estimate (38) is similar. 
16. Extension to systems of convection-diffusion-reaction problems

We may in a natural way directly extend the scope of methods and analysis to systems of convection-diffusion-reaction equations of the form

$$
\begin{cases}\dot{u}-\nabla \cdot(a \nabla u)+(b \cdot \nabla) u-f(u)=0 & \text { in } \Omega \times(0, T] \\ \partial_{n} u=0 & \text { on } \Gamma \times(0, T] \\ u(\cdot,)=u^{0} & \text { in } \Omega\end{cases}
$$

where $u=\left(u_{1}, \ldots, u_{d}\right)$ is a vector of concentrations, $a=a(x, t)$ is a diagonal matrix of diffusion coefficients, $b=b(x, t)$ is a given convection velocity, and $f(u)$ models reactions. Depending on the size of the coefficients $a$ and $b$ and the reaction term, this problem may exhibit more or less parabolic behavior, determined by the size of the strong stability factor coupled to the associated linearized dual problem (here linearized at the exact solution $u$ ):

$$
\begin{cases}-\dot{\phi}-\nabla \cdot(a \nabla \phi)-\nabla \cdot(\phi b)-\left(f^{\prime}(u)\right)^{\top} \phi=0 & \text { in } \Omega \times[0, T), \\ \partial_{n} \phi=0 & \text { on } \Gamma \times[0, T), \\ \phi(\cdot, T)=\psi & \text { in } \Omega,\end{cases}
$$

where $(\nabla \cdot(\phi b))_{i} \equiv \nabla \cdot\left(\phi b_{i}\right)$ for $i=1, \ldots, d$.

17. Examples of reaction-diffusion problems

We now present solutions to a selection of reaction-diffusion problems, including solutions of the dual backward problem and computation of stability factors.

\subsection{Moving heat source}

In Figures 10 and 11 we display mesh and solution at two different times for the adaptive $\mathrm{cG}(1) \mathrm{dG}(0)$ method applied to the the heat equation with a moving heat source producing a moving hot spot. We notice that the space mesh adapts to the solution.

\subsection{Adaptive time steps for the heat equation}

We consider again the heat equation, $\dot{u}-\Delta u=f$ with homogeneous Dirichlet boundary conditions on the unit square $(0,1) \times(0,1)$ over the time interval $[0,100]$. The source $f(x, t)=2 \pi^{2} \sin \left(\pi x_{1}\right) \sin \left(\pi x_{2}\right)\left[\sin \left(2 \pi^{2} t\right)+\cos \left(2 \pi^{2} t\right)\right]$ is periodic in time, with corresponding exact solution

$$
u(x, t)=\sin \left(\pi x_{1}\right) \sin \left(\pi x_{2}\right) \sin \left(2 \pi^{2} t\right) .
$$

In Figure 12 we show a computed solution using the $\mathrm{cG}(1) \mathrm{dG}(0)$ method, and we also plot the time evolution of the $L_{2}$-error in space together with the sequence of 

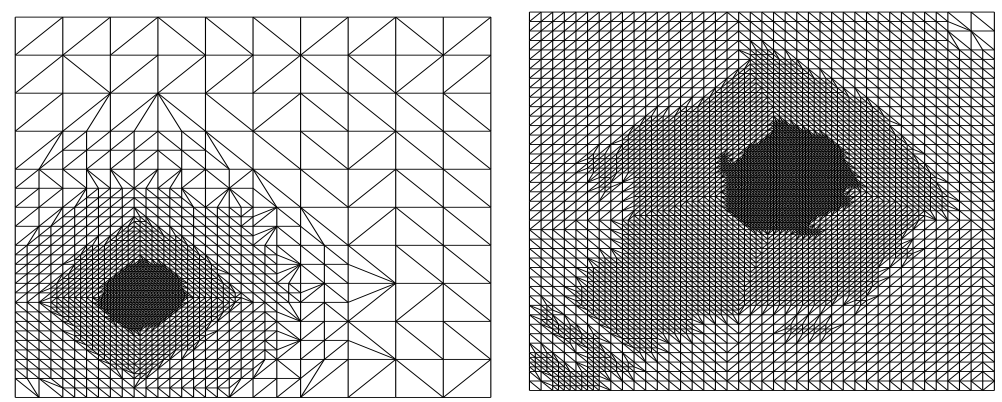

Figure 10. Meshes for moving source problem.
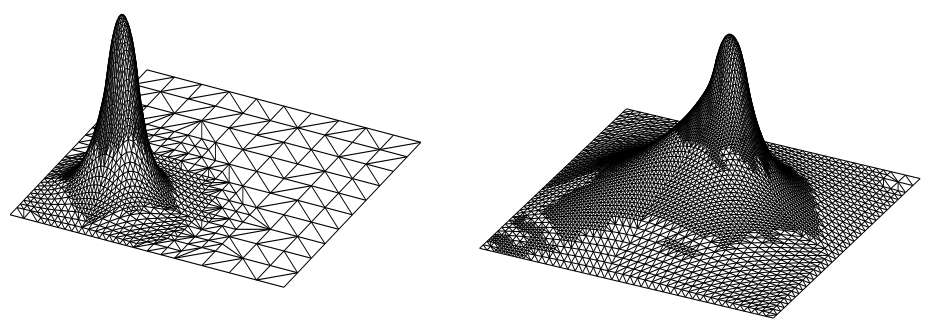

Figure 11. Solution for moving source problem.

adaptive time steps. We notice that the error does not grow with time, reflecting the parabolic nature of the problem. We also note the periodic time variation of the time steps, reflecting the periodicity of the solution, with larger time steps when the solution amplitude is small.

\subsection{Logistics reaction-diffusion}

We now consider the heat equation with a non-linear reaction-term, referred to as the logistics problem:

$$
\begin{cases}\dot{u}-\epsilon \Delta u=u(1-u) & \text { in } \Omega \times(0, T] \\ \partial_{n} u=0 & \text { on } \Gamma \times(0, T] \\ u(\cdot, 0)=u^{0} & \text { in } \Omega,\end{cases}
$$

with $\Omega=(0,1) \times(0,1), T=10, \epsilon=0.01$, and

$$
u^{0}(x)= \begin{cases}0, & 0<x_{1}<0.5 \\ 1, & 0.5 \leq x_{1}<1\end{cases}
$$

Encyclopedia of Computational Mechanics. Edited by Erwin Stein, René de Borst and Thomas J.R. Hughes. (c) 2004 John Wiley \& Sons, Ltd. 
$t=0$

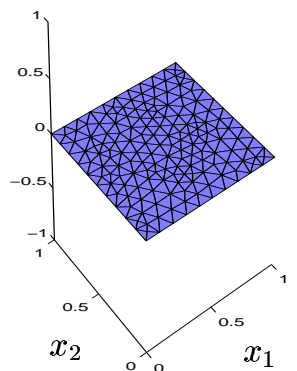

$t=37$

$T=100$

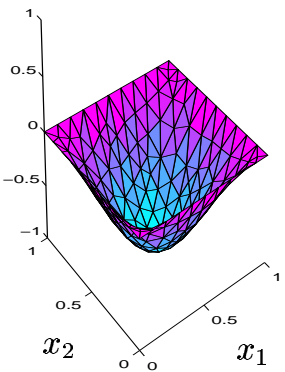

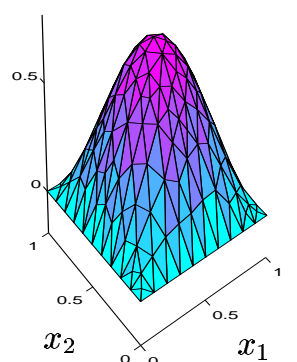
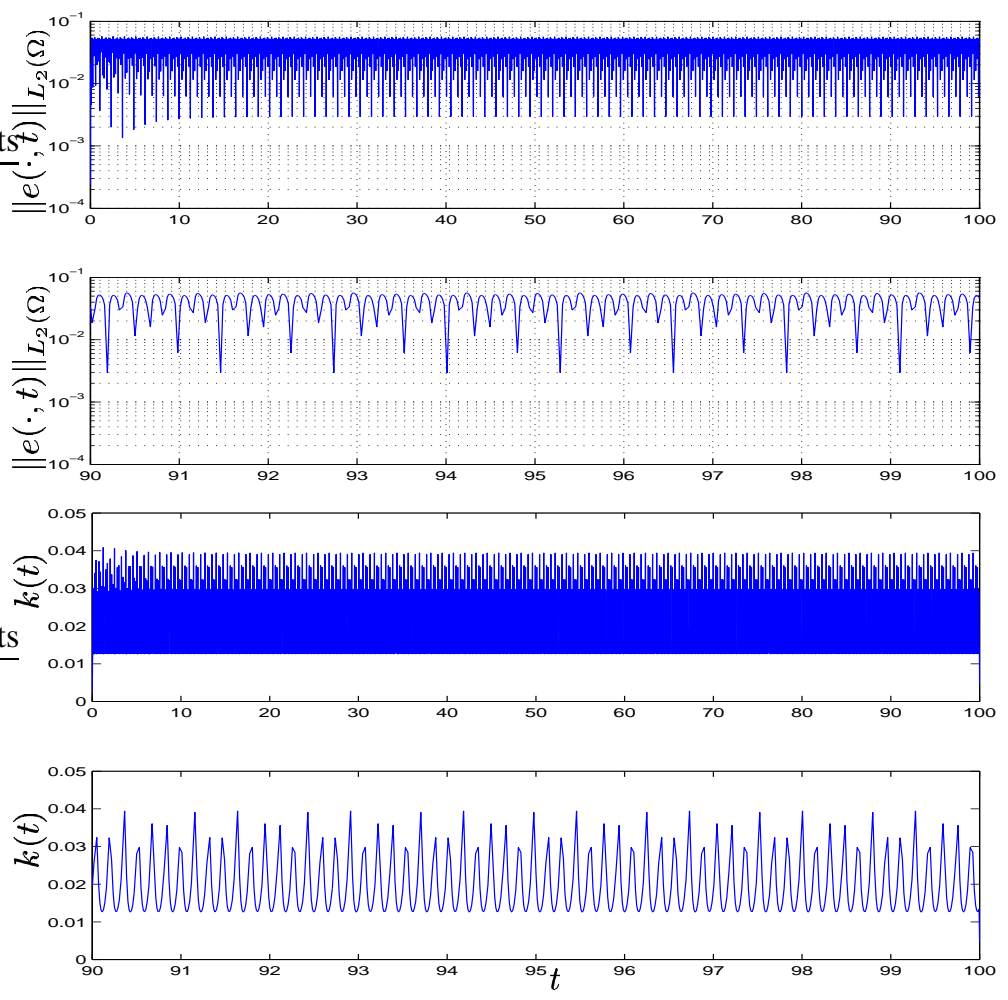

Figure 12. Heat equation: solution, error and adaptive time steps. 
Through the combined action of the diffusion and reaction the solution $u(x, t)$ tends to 1 for all $x$ with increasing time, see Figure 13. We focus interest at final time $T$ to a circle of radius $r=0.25$ centered at $x=(0.5,0.5)$. The corresponding dual problem linearized at the exact solution $u$ is given by

$$
\begin{cases}-\dot{\phi}-\epsilon \Delta \phi=(1-2 u) \phi & \text { in } \Omega \times[0, T) \\ \partial_{n} \phi=0 & \text { on } \Gamma \times[0, T) \\ \phi(\cdot, T)=\psi & \text { in } \Omega,\end{cases}
$$

where we choose $\psi=1 / \pi r^{2}$ within the circle and zero outside. In Figure 14 we plot the dual solution $\phi(\cdot, t)$ and also the stability factor $S_{c}(T, \psi)$ as function of $T$. As in the Akzo-Nobel problem discussed above, we note that $S_{c}(T, \psi)$ reaches a maximum for $T \sim 1$, and then decays somewhat for larger $T$. The decay with larger $T$ can be understood from the sign $(1-2 u)$ of the coefficient of the $\phi$-term in the dual problem, which is positive when $u(x, t)<0.5$ and thus is positive for $t$ small and $x_{1}<0.5$ and negative for larger $t$. The growth phase in $\psi(\cdot, t)$ thus occurs after a longer phase of decay if $T$ is large, and thus $S_{c}(T, \psi)$ may effectively be smaller for larger $T$, although the interval of integration is longer for large $T$.
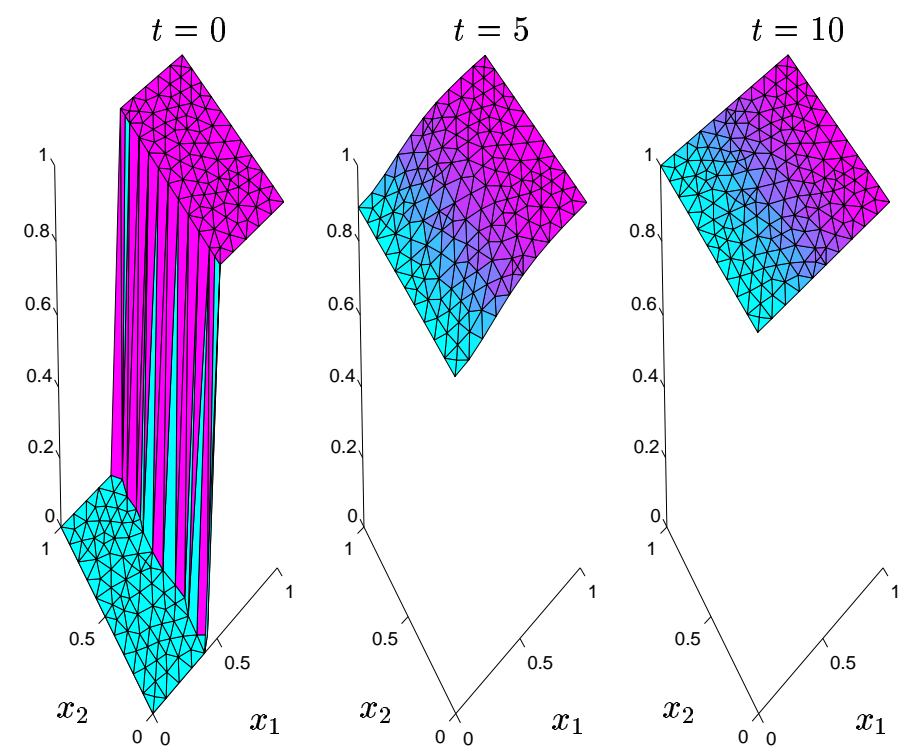

Figure 13. The logistics problem: solution at three different times. 
$t=0$
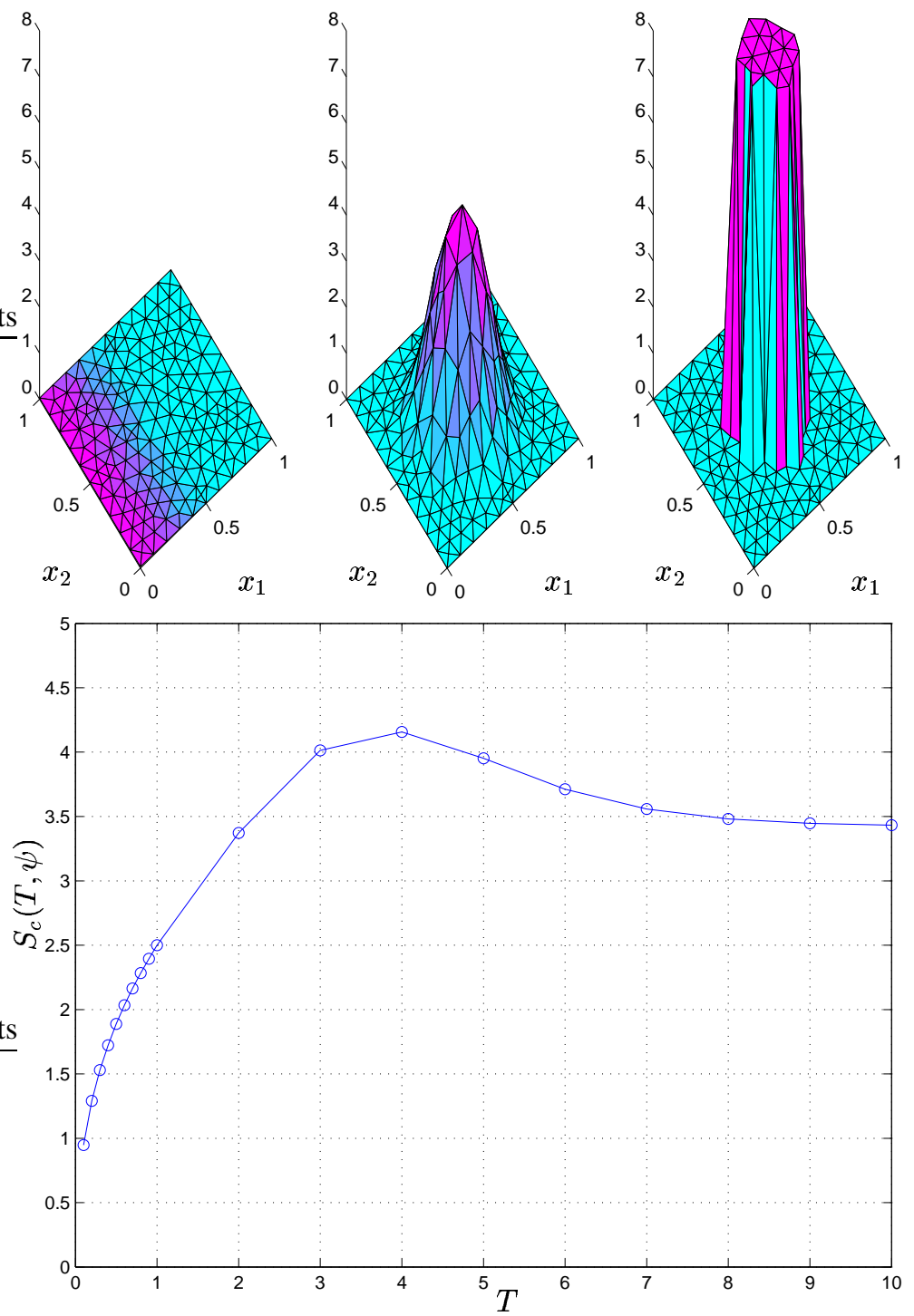

Figure 14. The logistics problem: dual solution and stability factor $S_{c}(T, \psi)$. 


\subsection{Moving reaction front}

Next, we consider a system of reaction-diffusion equations, modeling an auto-catalytic reaction where $A$ reacts to form $B$ with $B$ as a catalyst:

$$
A+2 B \rightarrow B+2 B \text {. }
$$

With $u_{1}$ the concentration of $A$ and $u_{2}$ that of $B$, the system takes the form

$$
\left\{\begin{array}{l}
\dot{u}_{1}-\epsilon \Delta u_{1}=-u_{1} u_{2}^{2}, \\
\dot{u}_{2}-\epsilon \Delta u_{2}=u_{1} u_{2}^{2},
\end{array}\right.
$$

on $\Omega \times(0,100]$ with $\Omega=(0,1) \times(0,0.25), \epsilon=0.0001$ and homogeneous Neumann boundary conditions. As initial conditions, we take

$$
u_{1}(x, 0)= \begin{cases}0, & 0<x_{1}<0.25 \\ 1, & 0.25 \leq x_{1}<1\end{cases}
$$

and $u_{2}(\cdot, 0)=1-u_{1}(\cdot, 0)$. The solution $u(x, t)$ corresponds to a reaction front, starting at $x_{1}=0.25$ and propagating to the the right in the domain until all of $A$ is consumed and the concentration of $B$ is $u_{2}=1$ in all of $\Omega$, see Figure 15 .
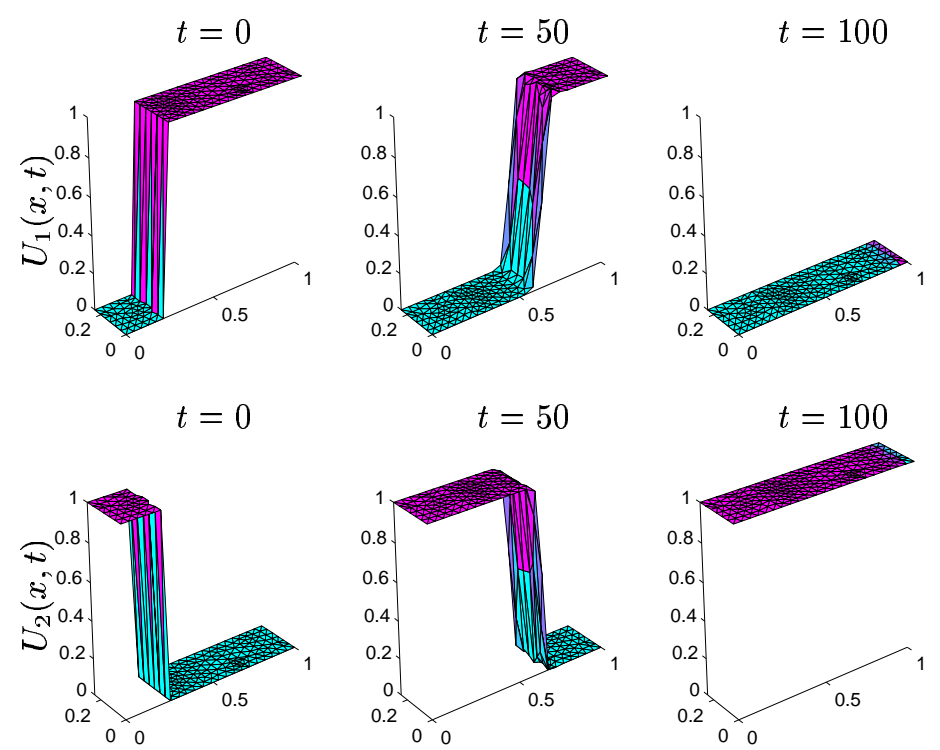

Figure 15. Reaction front problem: solution for the two components at three different times.

The dual problem, linearized at $u=\left(u_{1}, u_{2}\right)$, is given by

$$
\left\{\begin{array}{l}
-\dot{\phi}_{1}-\epsilon \Delta \phi_{1}=-u_{2}^{2} \phi_{1}+u_{2}^{2} \phi_{2} \\
-\dot{\phi}_{2}-\epsilon \Delta \phi_{2}=-2 u_{1} u_{2} \phi_{1}+2 u_{1} u_{2} \phi_{2}
\end{array}\right.
$$

Encyclopedia of Computational Mechanics. Edited by Erwin Stein, René de Borst and Thomas J.R. Hughes.

(c) 2004 John Wiley \& Sons, Ltd. 
As in the previous example, we take the final time data $\psi_{1}$ for the first component of the dual to be an approximation of a Dirac delta function centered in the middle of the domain, and $\psi_{2} \equiv 0$.

We note that the stability factor peaks at the time of active reaction, and that before and after the reaction front has swept the region of observation the stability factor $S_{c}(T, \psi)$ is significantly smaller.

$$
t=100
$$

$$
t=99
$$$$
t=0
$$
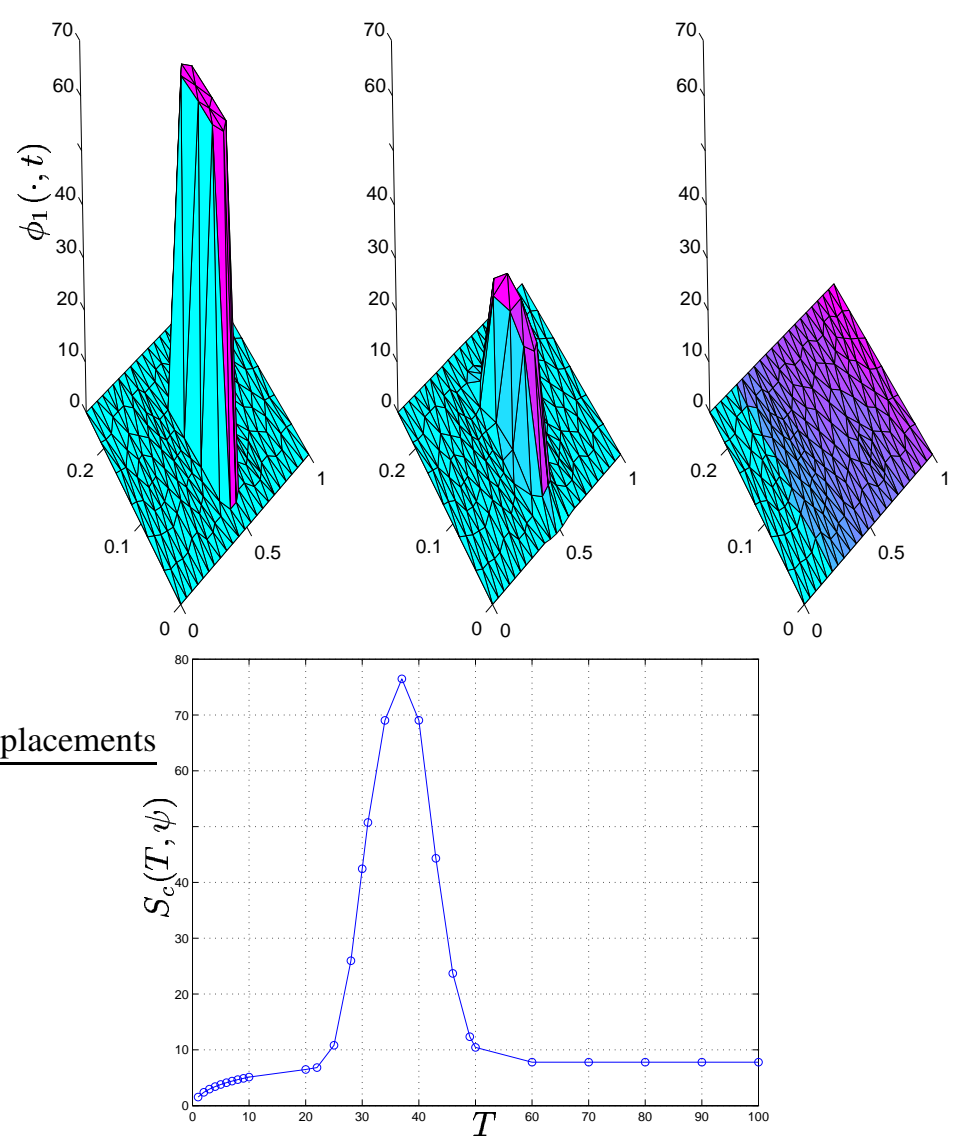

Figure 16. Reaction front problem: dual solution and stability factor $S_{c}(T)$ as function of $T$. 


\section{REFERENCES}

Eriksson K. and Johnson C. Adaptive Finite Element Methods for Parabolic Problems I: A Linear Model Problem. SIAM J. Numer. Anal. 1991; 28: 43-77.

Eriksson K. and Johnson C. Adaptive Finite Element Methods for Parabolic Problems II: Optimal Order Error Estimates in $L_{\infty} L_{2}$ and $L_{\infty} L_{\infty}$. SIAM J. Numer. Anal. 1995; 32: 706-740.

Eriksson K. and Johnson C. Adaptive Finite Element Methods for Parabolic Problems III: Time Steps Variable in Space. in preparation

Eriksson K. and Johnson C. Adaptive Finite Element Methods for Parabolic Problems IV: Nonlinear Problems. SIAM J. Numer. Anal. 1995; 32: 1729-1749.

Eriksson K. and Johnson C. Adaptive Finite Element Methods for Parabolic Problems V: Long-time integration. SIAM J. Numer. Anal. 1995; 32: 1750-1763.

Eriksson K., Johnson C. and Larsson S. Adaptive Finite Element Methods for Parabolic Problems VI: Analytic Semigroups. SIAM J. Numer. Anal. 1998; 35: $1315-1325$.

Eriksson K., Estep D., Hansbo P. and Johnson C. Introduction to Adaptive Methods for Differential Equations. Acta Numerica, Cambridge University Press 1995; $105-158$.

Eriksson K., Johnson C. and Thome V. Time Discretization of Parabolic Problems by the Discontinuous Galerkin Method. RAIRO MAN 1985; 19: 611-643.

Johnson C. Error Estimates and Adaptive Time-Step Control for a Class of One-Step Methods for Stiff Ordinary Differential Equations. SIAM J. Numer. Anal. 1988; 25: 908-926.

Logg A. Multi-Adaptive Galerkin Methods for ODEs I. Submitted to SIAM J. Sci. Comput.

Logg A. Multi-Adaptive Galerkin Methods for ODEs II: Implementation \& Applications. Submitted to SIAM J. Sci. Comput.

Eriksson K, Johnson C. and Logg A. Explicit Time-Stepping for Stiff ODEs. Submitted to SIAM J. Sci. Comput. 